\title{
A sequential inversion with outer iterations for the velocity and the intrinsic attenuation using an efficient wavefield inversion
}

\author{
Chao Song, Tariq Alkhalifah, Yuanyuan Li \\ King Abdullah University of Science and Technology (KAUST), \\ Physical Sciences and Engineering Division, \\ Thuwal, Saudi Arabia, 23955
}

(July 10, 2020)

Running head: A sequential viscoacoustic EWI

\begin{abstract}
Full-waveform inversion (FWI) has become a popular method to retrieve high-resolution subsurface model parameters. It is a highly non-linear optimization problem based on minimizing the misfit between the observed and predicted data. For intrinsically attenuating media, wave propagation experiences significant loss of energy. Thus, for better data fitting, it is crucial sometimes to consider attenuation in FWI. Viscoacoustic FWI aims at achieving a joint inversion of the velocity and attenuation models. However, multiparameter FWI imposes additional challenges including expanding the null space and facing parameter trade-off issues. Theoretically, an ideal way to mitigate the trade-off issue in multiparameter FWI is to apply the inverse Hessian operator to the parameter gradients. However, it is often not practical to calculate the full Hessian and its matrix inverse, as this will be extremely expensive. To improve the computational efficiency and mitigate the trade-off issue, we use an efficient wavefield inversion (EWI) method to invert for the velocity and the intrinsic attenuation. This approach is implemented in the frequency domain, and the velocity, in this
\end{abstract}


case, is complex valued in the viscoacoustic EWI. We propose a sequential update strategy for the velocity and the intrinsic attenuation, and we repeat the separate optimizations, we refer to as outer iterations, until the convergence is achieved. As viscoacoustic EWI is able to recover an accurate velocity model, the velocity update leakage to the $Q$ model is largely reduced. We show the effectiveness of this approach using synthetic data generated for the viscoacoustic Marmousi and Overthrust models. To further demonstrate the validity of the proposed approach, we generate data in the time domain using a viscoelastic wave equation solver, and obtain reasonable inversion results in the frequency domain using the viscoacoustic approximation. 


\section{INTRODUCTION}

The Earth is generally anelastic and seismic waves will face dispersion and attenuation effects as they propagate (Aki and Richards, 2002), especially in regions with gas clouds and mud channels. An accurate description of the attenuation model will help us compensate for the energy loss and phase shifts in a $Q$-compensation reverse time migration (QRTM) or a least-squares RTM (QLSRTM) (Zhu et al., 2014; Dutta and Schuster, 2014). Seismic signals attenuate faster as the frequency increases, and the peak frequency will reduce during wave propagation. This reduction can be used to invert for the attenuation model tomographically (Quan and Harris, 1997; Hu et al., 2011). However, these methods rely highly on the accuracy of the velocity model. Thus, inversions for both the velocity model and the attenuation model are required.

Full-waveform inversion (FWI) aims at retrieving physical properties of the subsurface by minimizing the misfit between the observed and predicted data (Tarantola, 1984). As the observed data are influenced by a variety of subsurface parameters including velocity, density, anisotropy, and intrinsic attenuation, FWI has the potential to recover these parameters, but with different sensitivities and tradeoff. With the help of the viscoacoustic assumption, FWI can provide inversion results for both the velocity and the attenuation models (Kamei and Pratt, 2008; Malinowski et al., 2011; Gao and Wang, 2016). However, multiparameter FWI has always been a challenge, because parameter update tradeoff may cause inaccurate inversion results (Tarantola, 1994). This trade-off issue is more severe for the viscoacoustic case, as the velocity and the intrinsic attenuation share similar radiation patterns. The velocity and the intrinsic attenuation are both independent of the scattering angle with varying magnitude of data sensitivities. Suggestions to balance the sensitivities 
of the velocity and the intrinsic attenuation in a simultaneous inversion have been proposed (Malinowski et al., 2011; Gao and Wang, 2016). Simultaneous inversion calculates the gradients of different parameters using the same background models, and updates all the parameters at once in every iteration. The inverse Hessian operator can resolve part of the trade-off problem, at least theoretically (Kamei and Pratt, 2013; Métivier et al., 2013), but it is not practical to use due to its cost. A preconditioned truncated Newton method was proposed to resolve the trade-off issue between parameters (Yang et al., 2018). Methods mentioned above are mostly based on using the velocity and intrinsic attenuation gradients with respect to the objective function. However, unconstrained waveform inversion methods based purely on gradients struggle the most with null-space issues. da Silva et al. (2019) introduce a semiglobal viscoacoustic FWI, and they perform a joint estimation of P-wave velocity and intrinsic attenuation. This method uses local iterations to update velocity only, nested within a loop of global iterations for the intrinsic attenuation.

Previous work on using a simultaneous inversion for the velocity and the intrinsic attenuation using FWI has shown promise. However, joint multiparameter inversion is often affected by crosstalk. While the sequential inversion strategy can get reasonable velocity and intrinsic attenuation inversion results (Kamei and Pratt, 2008). However, the nonlinearity in multiparameter waveform inversion is still a great challenge. To resolve this matter, we propose to update the velocity and the intrinsic attenuation sequentially with outer iterations using a recently developed method, which is referred to as efficient wavefield inversion (EWI). EWI is an implementation of wavefield reconstruction inversion (WRI) in which a modified source function is used as an independent parameter to absorb the parameter perturbations (Van Leeuwen and Herrmann, 2013; Alkhalifah and Song, 2019). Inner iterations between calculating the wavefield and the modified source function add multiscat- 
tering components to the reconstructed wavefield. Using a direct division (deconvolution) method, the medium parameters can be calculated directly. In multiparameter waveform inversion, EWI demonstrates its effectiveness in recovering a good velocity model, so a sequential inversion for any other parameter will suffer less from the cross-talk issue (Song et al., 2019a; Song and Alkhalifah, 2020a). For viscoacoustic media, as the velocity is the dominant parameter controlling the wave propagation, and it has a larger influence on the data, we first update the velocity model through the selected frequency band (Gao and Wang, 2016). Then, we keep the updated velocity fixed, and then update the attenuation model. As the attenuation model is improved, the velocity optimization convergence will be accelerated (Xue et al., 2017). Finally, we use outer iterations (over the full range of frequencies) to repeat these two steps. The inverted intrinsic attenuation parameter is often highly oscillatory (Kamei and Pratt, 2013). So we apply an iterative total variation (TV) denoising to improve the inverted attenuation model in each outer iteration. TV denoising is a powerful tool to smooth out the oscillatory artifacts and impose the sharp edges into the attenuation model.

In this paper, we start with a brief introduction of joint viscoacoustic FWI. In the theory section, we explain how we use viscoacoustic EWI to perform a multiparameter inversion for viscoacoustic media in a sequential matter. We use a toy Gaussian anomaly model to show the advantage of the sequential inversion with outer iterations scheme over the simultaneous inversion. Finally, applications on viscoacoustic Marmousi and Overthrust models show that the proposed approach can yield reasonable inversion results for viscoacoustic media. 


\section{THEORY}

To simulate wave propagation considering intrinsic attenuation of energy inside the Earth, we use a viscoacoustic wave equation with a constant density expressed in the frequency domain (Blanch et al., 1995; Malinowski et al., 2011; Gao and Wang, 2016).

$$
\frac{\omega^{2}}{\widetilde{v}^{2}} u(\mathbf{x}, \omega)+\nabla^{2} u(\mathbf{x}, \omega)=f\left(\mathbf{x}, \mathbf{x}_{\mathbf{s}}, \omega\right)
$$

where $\omega$ is the angular frequency, $u(\mathbf{x}, \omega)$ denotes the pressure wavefield, and $f\left(\mathbf{x}, \mathbf{x}_{\mathbf{s}}, \omega\right)$ is the source function. $\mathbf{x}_{\mathbf{s}}$ represents the spatial source coordinates. $\widetilde{v}$ denotes the complexvalued wave speed with an intrinsic attenuation coefficient embedded in it, as follows (Toksöz and Johnston, 1981):

$$
\widetilde{v}=v\left[1-\frac{i}{2 Q}\right]
$$

where $v$ is the velocity, $Q$ is the quality factor that describes the intrinsic attenuation in viscoacoustic media, and the higher $Q$, the less an intrinsic attenuation. However, this viscoacoustic wave equation ignores the dispersion effect of the signal, and it only depicts the amplitude aspect of attenuation. This will not be a problem in estimating the intrinsic attenuation as we will use a rather narrow frequency band in the inversion. $v$ is the wave speed of compressional waves, and $i=\sqrt{-1}$. If we use $m$ to represent the squared complexvalued slowness $\frac{1}{\widetilde{v}^{2}}$, and $c=\frac{1}{\left[1-\frac{i}{2 Q}\right]^{2}}$, equation 1 can be written as:

$$
\omega^{2} m c u(\mathbf{x}, \omega)+\nabla^{2} u(\mathbf{x}, \omega)=f\left(\mathbf{x}, \mathbf{x}_{\mathbf{s}}, \omega\right) \text {. }
$$

The frequency-domain viscoacoustic wave equation can be expressed in compact form as:

$$
\mathbf{L}(\omega, m, c) u(\mathbf{x}, \omega)=f\left(\mathbf{x}, \mathbf{x}_{\mathbf{s}}, \omega\right),
$$

where, $\mathbf{L}(\omega, m, c)=\omega^{2} m c+\nabla^{2}$ is the impedance matrix, which is also referred to as the modeling operator. Usually, the sparse impedance matrix $\mathbf{L}$ is decomposed into a 
lower part $(L)$ and an upper part $(U)$ using LU decomposition (Pratt et al., 1998; Pratt, 1999). As $\mathbf{L}(\omega, m, c)$ is independent of the source function, the stored $L$ and $U$ matrices can be applied on many sources for efficient wavefield calculation. Usually, the cost of the $\mathrm{LU}$ decomposition is affordable for $2 \mathrm{D}$ problems. However, larger models like for 3D experiments require significantly more RAM for storage and increases the computational cost (Wang et al., 2011; Amestoy et al., 2016). In the conventional FWI implementation, we tend to measure the misfit between the observed and predicted data under the constraint of the wave equation:

$$
J(m, c)=\frac{1}{2} \sum_{j}\left\|d_{j}-C u_{j}\right\|_{2}^{2} \quad \text { s.t. } \quad \mathbf{L}(\omega, m, c) u_{j}=f_{j},
$$

where, $j$ is the source index, and $d_{j}$ is the recorded data. $C$ is an operator mapping the wavefield onto the sensor locations. Based on the adjoint-state method (Plessix, 2006), the $m$ and $c$ gradients are given by the cross-correlation between the background and adjoint wavefields.

$$
\nabla_{p} J=-\Re \sum_{j}\left\{\omega^{2} \frac{\partial \mathbf{L}}{\partial p} u_{j} \mathbf{L}^{-T}\left(C^{T} \Delta d_{j}^{*}\right)\right\},
$$

where $p$ is the target parameter representing the $m$ or $c$. The gradients of the velocity and $Q$ models in FWI are shown in Malinowski et al. (2011). T denotes ordinary matrix transpose, and the superscript $*$ represents the complex conjugate. $\Delta d_{j}=d_{j}-C u_{j}$ represents the data residual. The adjoint wavefield is calculated by back propagating the complex conjugate form of the data residual $\Delta d_{j}^{*}$ at the sensors locations. Conventional FWI requires a large computational cost and memory in storing the background wavefields or at least one of them. The wavefield $u_{j}$ is calculated using the wave equation, which depends on media parameters. This dependency causes the non-linearity of the objective function. In multiparameter waveform inversion, we have another level of complexity. Since the data residuals could be 
caused by different media parameter perturbations, gradient-based optimization methods will suffer from the trade-off issue between different parameters.

\section{Efficient wavefield inversion for viscoacoustic media}

To mitigate the classic trade-off problem, we use EWI for the multiparameter inversion in viscoacoustic media with a sequential update scheme. The objective function of EWI is given by:

$$
E\left(u_{j}, f_{e j}\right)=\frac{1}{2} \sum_{j}\left\|d_{j}-C u_{j}\right\|_{2}^{2}+\frac{\alpha^{2}}{2} \sum_{j}\left\|\mathbf{L}_{\mathbf{0}} u_{j}-f_{e j}\right\|_{2}^{2},
$$

where $\alpha$ is a weighting parameter balancing the priority between data fitting and satisfying the background wave equation. At the beginning of the inversion process, we start with a background modeling operator $\mathbf{L}_{\mathbf{0}}\left(\omega, m_{0}, c_{0}\right)=\omega^{2} m_{0} c_{0}+\nabla^{2}$, which corresponds to the background medium parameters: $m_{0}$ and $c_{0}$. In equation 7 , the modified source function $f_{e j}$ starts with the original source function $f_{j}$ in the inversion process. To calculate the wavefield, which needs to simultaneously satisfy the data fitting objective and the wave equation, we minimize $E$ by solving $\nabla_{u} E=0$. Thus, the wavefield satisfies the following linear equation:

$$
\left(\begin{array}{c}
\alpha \mathbf{L}_{0} \\
C
\end{array}\right) u_{j}=\left(\begin{array}{c}
\alpha f_{e j} \\
d_{j}
\end{array}\right)
$$

This background wavefield will include only single scattered energy from the missing perturbations, and such energy often dominates the wavefield, compared to multiscattering. The modified source function that satisfies the wave equation can be calculated using background operator $\mathbf{L}_{\mathbf{0}}$ as:

$$
f_{e j}=\mathbf{L}_{\mathbf{0}} u_{j}
$$


In equation $9, \mathbf{L}_{\mathbf{0}}$ is updated as a consequence of updating the background models. If we perturb parameters $m, c$ by $\delta m, \delta c$, then $m$ and $c$ can be defined as:

$$
\begin{gathered}
m=m_{0}+\delta m, \\
c=c_{0}+\delta c .
\end{gathered}
$$

We insert equations 10 and 11 into the modeling operator $\mathbf{L}(\omega, m, c)=\omega^{2} m c+\nabla^{2}$, we obtain

$$
\begin{aligned}
& \mathbf{L}(\omega, m, c)=\omega^{2} m c+\nabla^{2}=\omega^{2}\left(m_{0}+\delta m\right)\left(c_{0}+\delta c\right)+\nabla^{2} \\
& =\mathbf{L}_{\mathbf{0}}\left(\omega, m_{0}, c_{0}\right)+\omega^{2} \delta m c_{0}+\omega^{2} \delta c m_{0}+\omega^{2} \delta c \delta m .
\end{aligned}
$$

According to the relationship between the background and true viscoacoustic modeling operators shown in equation 11, we can derive the relationship between the original and modified source functions. The frequency-domain wave equation $\mathbf{L} u_{j}=f_{j}$ is equivalent to

$$
\begin{aligned}
& \left(\mathbf{L}_{\mathbf{0}}+\omega^{2} \delta m c_{0}+\omega^{2} m_{0} \delta c+\omega^{2} \delta m \delta c\right) u_{j}=f_{j},<=> \\
& \mathbf{L}_{\mathbf{0}} u_{j}=f_{j}-\omega^{2}\left(\delta m c_{0}+m_{0} \delta c+\delta m \delta c\right) u_{j}=f_{e j} .
\end{aligned}
$$

If we ignore the second-order approximation term $\omega^{2} \delta m \delta c u$, we finally get

$$
f_{e j}=f_{j}-\omega^{2}\left(\delta m c_{0}+m_{0} \delta c\right) u_{j}
$$

Equation 14 shows that the modified source function $f_{e}$ contains the squared slowness perturbation and the attenuation perturbation, and they will act as secondary sources to generate multiscattering information during the inner iterations between equations 8 and 9 . In our implementation of viscoacoustic EWI, we sequentially invert for the velocity first, and 
the intrinsic attenuation secondly. It is natural to update the velocity first, as velocity is the dominant parameter controlling the wave propagation. In this two-stage update scheme, when we update one target parameter, we assume the other parameter perturbation is zero. We repeat these two stages using EWI with outer iterations, and we refer to this inversion scheme for the velocity and $Q$ as a sequential viscoacoustic EWI. According to equation 14, $\delta m$ and $\delta c$ can be calculated using:

$$
\begin{gathered}
\delta m=\sum_{j} \frac{\left(u_{j} c_{0}\right)^{*}\left(f_{j}-f_{e j}\right)}{\omega^{2}\left(u_{j} c_{0}\right)\left(u_{j} c_{0}\right)^{*}+\lambda_{1}}, \\
\delta c=\sum_{j} \frac{\left(u_{j} m_{0}\right)^{*}\left(f_{j}-f_{e j}\right)}{\omega^{2}\left(u_{j} m_{0}\right)\left(u_{j} m_{0}\right)^{*}+\lambda_{2}},
\end{gathered}
$$

where, $\lambda_{1}$ and $\lambda_{2}$ are small values to avoid dividing over zero. Usually, we use one percentage of the maximum values of the denominators in equations 15 and 16. After calculating the parameter perturbations $\delta m$ and $\delta c$, we update the velocity and $Q$ background models until the convergence is achieved. Finally, the velocity and $Q$ models are evaluated using:

$$
\begin{gathered}
v=\Re \sqrt{\frac{1}{m_{0}}}, \\
Q=\Im \frac{1}{2\left(1-\sqrt{1 / c_{0}}\right)},
\end{gathered}
$$

where $\Re$ and $\Im$ mean taking the real and imaginary parts of complex values. The velocity and the quality factor $Q$ both have isotropic radiation patterns, and $Q$ has a lower sensitivity than the velocity in the data for all scattering angles (da Silva et al., 2019). Thus, if we start the $Q$ inversion with a poor initial velocity model, the velocity error will affect the $Q$ inversion result. As parameter perturbations include multiscattering components, the convergence rate in each parameter inversion accelerates (Alkhalifah and Wu, 2016; Song et al., 2019b). 


\section{Total variation denoising}

The inverted $Q$ model is usually contaminated by high-wavenumber oscillations. Considerable regularizations and smooth filtering are recommended to apply to the intrinsic attenuation parameter (Kamei and Pratt, 2013). Total variation (TV) regularization is often used to preserve sharp edges without oversmoothing the reconstructed model (Kalita et al., 2019). We use an iterative total variation smoothing method to remove the highwavenumber oscillations in the inverted $Q$ model (Rudin et al., 1992; Vogel and Oman, 1996). Consider the inverted $Q$ model is given by:

$$
Q=Q_{t v}+\epsilon
$$

where $Q_{t v}$ represents the desired clean attenuation model after TV denoising, $\epsilon$ represents the undesired oscillations, and $Q$ represents the originally inverted attenuation model. We want to isolate $Q_{t v}$ from $Q$, so we use the minimization of the TV-penalized least-squares objective function, as follows:

$$
E\left(Q_{t v}\right)=\frac{1}{2}\left\|Q_{t v}-Q\right\|_{2}^{2}+\beta J_{\mu}\left(Q_{t v}\right)
$$

where

$$
J_{\mu}\left(Q_{t v}\right)=\int_{\Omega} \sqrt{\left|\nabla Q_{t v}\right|_{2}^{2}+\mu} d \mathbf{x} .
$$

In equation $21, \nabla Q_{t v}=\left(\frac{\partial Q_{t v}}{\partial x}, \frac{\partial Q_{t v}}{\partial z}\right) . \mu$ is a small real positive value number, and $\beta$ controls the weight of the total variation. We use $g\left(Q_{t v}\right)$ to denote the gradient with respect to $Q_{t v}$ of the equation 20 , and it is given by:

$$
g\left(Q_{t v}\right)=Q_{t v}-Q+\beta \nabla \cdot\left(\frac{\nabla Q_{t v}}{\sqrt{\left|\nabla Q_{t v}\right|_{2}^{2}+\mu}}\right) .
$$


Finally, we use a gradient descent scheme to update $Q_{t v}$ until we achieve convergence. It is given by:

$$
Q_{t v}^{(k+1)}=Q_{t v}^{(k)}-\tau_{k} g\left(Q_{t v}^{(k)}\right), \quad k=0,1,2 \ldots
$$

where $k$ is the $k$-th iteration, and $\tau_{k}$ is the step length. We use $\tau_{k}=0.2$ and $\beta=0.1$ in all the examples shown in this paper, based on trial and error. This update scheme is a linear process, which only requires little extra cost. The algorithm of our sequential viscoacoustic EWI is shown in Algorithm 1.

\section{NUMERICAL EXAMPLES}

\section{Gaussian anomaly model}

We include a Gaussian anomaly velocity on the right-hand side of a homogeneous background, and a Gaussian anomaly $Q$ on the left-hand side of a homogeneous background, as shown in Figures 1a and 1b, respectively. A full aperture acquisition is used with 36 sources and 400 receivers along all the boundaries of the model. The source locations are denoted by the $*$ symbols in Figure 1a. The selected frequency band extends from $3 \mathrm{~Hz}$ to $6 \mathrm{~Hz}$ with $0.5 \mathrm{~Hz}$ sampling interval. We start the inversion from low frequencies. We generate the synthetic data in the frequency domain using a delta function source. For all the numerical examples shown in this paper, the forward operator uses absorbing boundary conditions without free surface. The coefficient $\alpha^{2}$ we use in all examples in this paper is $10^{7}$, and we choose this value based on previous trial and error tests.

The initial velocity and $Q$ models used in the inversion are the homogeneous background models. We use two inner iterations within each frequency (for all the examples in this paper). We first use a joint viscoacoustic EWI for the velocity and the $Q$ model. In the 
joint inversion scheme, we use Algorithm 1 without the second sweep, and just update the velocity and the $Q$ models simultaneously in the first frequency band sweep. After ten outer iterations sweeping the whole frequency band in each iteration, the inverted velocity is shown in Figure 2a. We perform a sequential inversion with outer iterations for the velocity and then we invert for the $Q$ model using the same inversion setup. In the sequential viscoacoustic EWI, and after updating the velocity, we begin to invert for the $Q$ model. We define these two steps as one outer iteration. After ten outer iterations (sweeping the whole frequency band from low to high ten times), the inverted velocity is shown in Figure 2b. The difference between the inverted velocity models using the joint and sequential viscoacoustic EWI appears to be small. When we show the differences between the true and inverted velocities in Figure 3, we observe that the sequential viscoacoustic EWI inverted velocity has lower error than that from the joint viscoacoustic EWI. This more accurate velocity can help mitigate the trade-off issue. The inverted $Q$ models using joint and sequential viscoacoustic EWI are shown in Figures 4a and 4b, respectively. The results show that the joint viscoacoustic EWI can recover the main anomaly of the $Q$ model. However, there is a clear footprint of the velocity update in the $Q$ model. By comparison, the sequential viscoacoustic EWI can invert for a clean $Q$ model without influence from the velocity update leakage. Finally, we show in Figure 5 the normalized data misfit of the joint and sequential viscoacoustic EWI, and we can observe that the sequential inversion strategy achieves faster convergence than the joint one.

\section{D Marmousi model}

We next apply the proposed method on a viscoacoustic Marmousi model. The true velocity model is shown in Figure 6a, and we smooth the true model three times with a Gaussian 
window size of $1000 \times 1000 \mathrm{~m}$ to obtain the initial velocity, as shown in Figure $6 \mathrm{~b}$. The size of the model is $461 \times 284$, and the spatial sampling interval in both vertical and horizontal directions is $25 \mathrm{~m}$. The true $Q$ model is shown in Figure 7, and the initial $Q$ model is homogeneous with a high value of 100 .

We use 23 sources uniformly distributed on the surface, and their locations are given by * symbols in Figure 6a. All the grid points on the surface act as receivers. We implement the proposed method in the frequency domain, and the selected frequency band extends from 3 $\mathrm{Hz}$ to $10 \mathrm{~Hz}$ with $0.5 \mathrm{~Hz}$ sampling interval. The synthetic data are generated from a delta source function for all the selected frequencies using the true velocity and $Q$ models. We keep the thin water layer on the surface in the models fixed in all the inversion implementations. We first use an acoustic FWI and an acoustic EWI to invert for the velocity. After 50 outer iterations, the inverted velocity models are shown in Figures 8a and 8b, respectively. As the intrinsic attenuation effect is ignored using a pure acoustic approximation, acoustic FWI fails to recover the detailed structures of the true model, especially in the deep area. Although acoustic EWI improves the inversion result a lot, the regions where the high intrinsic attenuation exists are still not well inverted, especially in the circled area. We then use viscoacoustic EWI to invert for the velocity with the fixed $Q$ model. After the same number of outer iterations, the inverted velocity model is shown in Figure 9a. When we include the $Q$ in the inversion process, the inverted velocity and $Q$ models using a sequential inversion are shown in Figures 9b, and 10, respectively. Figure 10 shows that the proposed method can recover a reasonably good $Q$ model, and almost no velocity update leakage in the $Q$ model. This well reconstructed $Q$ model helps us get a more accurate velocity inversion result. As the intrinsic attenuation (low $Q$ ) is only located in the middle area of the model, wavefield amplitude is only affected in the deep parts. As a result, the 
inverted velocity models with or without considering $Q$ model looks similar in the shallow part, and there are only different in the deep part (as the black arrows point out). This is more obvious looking at two profiles at $3.75 \mathrm{~km}$ and $8.25 \mathrm{~km}$, which are shown in Figures 11a and $11 \mathrm{~b}$, respectively. It is obvious that the inverted velocity models using viscoacoustic EWI are closer to the true velocity model. The meaning of an accurate $Q$ inversion is more clearly revealed in the $Q$ compensation least-squares reverse time migration (QLSRTM). The QLSRTM image for the initial velocity and the background $Q$ is shown in Figure 12a. We observe that the events in the shallow part are not well focused due to the error in the velocity. The events in the deep part are blurry and weak because of the presence of the high intrinsic attenuation at the mid-range depth. Using the inverted velocity in Figure 9a and the background $Q$, the QLSRTM image is shown in Figure 12b. As the velocity improves, the image quality improves as well. It is not as good as the QLSRTM image corresponding to the inverted velocity and $Q$ models using viscoacoustic EWI, as shown in Figure 12c. As we reasonably recovered the $Q$ model at the correct position, the amplitude of the events in the deep part are enhanced (as the red arrows point out).

The inverted $Q$ model has many oscillatory artifacts as we expected. To further improve the inverted $Q$ model, we apply an iterative total variation (TV) denoising to the updated $Q$ model in each outer iteration. With a TV constraint applied to the $Q$ model, the inverted velocity and $Q$ are shown in Figures $13 \mathrm{a}$ and $14 \mathrm{a}$, respectively. It is clear that the TV regularization helps preserve the sharp edges of the $Q$ model and eliminate the artifacts. By comparison, we show the sequential inversion results of viscoacoustic FWI using the same inversion setup in Figures 13b and 14b, respectively. The viscoacoustic FWI uses the velocity and the intrinsic attenuation gradients shown in equation 6 to update the model, and the inverted velocity is generally good. However, it is not as good as the inverted 
velocity using viscoacoustic EWI, especially in the deep part. The inverted $Q$ using FWI is obviously wrong, and it comes from the velocity update leakage. Both viscoacoustic FWI and viscoacoustic EWI use a sequential inversion scheme for the velocity and $Q$ models, but EWI does a better job in reducing the trade-off problem. This is because viscoacoustic EWI recovers a more accurate velocity, in this case, very limited velocity leaks to the $Q$ model. As viscoacoustic FWI fails to recover a good velocity with a limited number of iterations, velocity leakage to the $Q$ model is still severe. By comparison, we implement a joint viscoacoustic EWI for the velocity and the intrinsic attenuation, and the results are shown in Figures 13c and 14c, respectively. It is clear that the inverted $Q$ model using a joint inversion scheme suffers from the velocity leakage, especially in the shallow area. As the arrows point out in Figures 13a-13c, the sequential viscoacoustic EWI yielded the best velocity result among the methods used above. Finally, we show the percent root-meansquare (RMS) errors in the inverted velocity models per outer iteration measured with respect to the true Marmousi velocity model in Figure 15. Clearly, both the acoustic and viscoacoustic FWI and EWI will generally converge. Since FWI is prone to cycle skipping, EWI clearly converges faster than FWI, regardless of the used inversion scheme. Compared to the joint viscoacoustic EWI, the sequential viscoacoustic EWI reaches a lower velocity model RMS error. We also show in Figure 16 RMS errors for the inverted $Q$ models per outer iteration. We can clearly see that the sequential viscoacoustic EWI has the best convergence properties for the $Q$ model inversion.

\section{D Overthrust model}

We further consider another example given by the viscoacoustic Overthrust model. The true and initial velocity models are shown in Figures $17 \mathrm{a}$ and $17 \mathrm{~b}$, respectively. The initial 
velocity is obtained by applying a Gaussian smoother on the true model three times with the same window size as the previous example. The size of the model is $401 \times 100$ with $25 \mathrm{~m}$ spacing in both directions. The true and initial $Q$ models are shown in Figures 18a and 18b, respectively. The strongly attenuating (low $Q$ ) area in this model is located in the shallow part, and the initial $Q$ model is homogeneous with a high value of 100 . In this example, we use a Ricker wavelet with a dominant frequency of $10 \mathrm{~Hz}$ to generate the synthetic data.

In this inversion setup, we use 20 sources on the surface with a uniform spatial interval of $0.5 \mathrm{~km}$ (demonstrated by $*$ symbols in Figure 17a), and we use all the grid points on the surface as receivers. The selected frequency band is from $3 \mathrm{~Hz}$ to $15 \mathrm{~Hz}$ with $0.5 \mathrm{~Hz}$ sampling interval. We use the same inversion strategy as the previous the example, sweeping though all the selected frequencies for velocity first, then for a $Q$ model. We perform a viscoacoustic EWI to invert for the velocity and the $Q$ model, and the inverted velocity and $Q$ models are shown in Figures 19a and 20a, respectively. We observe that the inverted velocity and the $Q$ models are very well recovered with limited trade-off. As we apply TV denoising to the inverted $Q$ model, the inverted $Q$ model becomes free of oscillations with sharp boundaries. In Figure 20a, there are mild artifacts on the right-hand side, which cannot be removed by the TV denoising. These weak artifacts maybe a result of the velocity leakage. The inversion results of the conventional viscoacoustic FWI using the same inversion setup are shown in Figures $19 \mathrm{~b}$ and $20 \mathrm{~b}$, respectively. They are inferior to what we obtained in the viscoacoustic EWI. The inverted $Q$ model in Figure 20b suffers from the velocity leakage. To test the robustness of the proposed method for noisy data, we add Gaussian noise resulting in signal-to-noise ratio (SNR) of $10 \mathrm{db}$ in the synthetic data. We show the real, and imaginary parts of the clean and noisy data for a source located in the middle at $5 \mathrm{~Hz}$ in Figures 21a and 21b, respectively. With the same inversion setup, the viscoacoustic 
EWI inverted velocity, and $Q$ models using noisy data are shown in Figures 22a and 22b, respectively. Using the noisy data, the inverted velocity contains slight oscillations, which are almost negligible. The noise added to the data mainly affect the amplitude, and mildly affect the phase information. The velocity inversion depends on both amplitude and phase information. Thus, even though the amplitudes in the data are contaminated with noise, the phase information can provide reliable velocity inversion information. However, the attenuation model is mainly retrieved from the amplitude information. As a result, the inverted $Q$ model is more vulnerable to noise in the data, and thus, the inverted $Q$ model contains slightly higher errors.

To further demonstrate the effectiveness of the proposed method using an independent modeling code, we generate the observed data using a time-domain (instead of frequency domain) viscoacoustic wave equation. A shot gather is shown in Figure 23a. For a even more realistic test, we simulate observed data using a time-domain viscoelastic modeling with an explosive source (Robertsson et al., 1994). We generate an S-wave velocity model by dividing the $\mathrm{P}$-wave velocity by $\sqrt{3}$. For the density, we use a constant value of $1.5 \mathrm{~kg} / \mathrm{m}^{3}$. The Swave attenuation model $Q_{s}$ is the same as the model for the $\mathrm{P}$ wave. We use a time-domain Ricker wavelet to generate the synthetic data, and one shot gather of the z-component data is shown in Figure 23b. Clearly, the data generated from the viscoelastic wave equation contain S-wave arrivals (as shown in the circled area). We perform viscoacoustic EWI using the same inversion setup as we used before for this model. In this case, we use a viscoacoustic wave equation with variable density to resolve the expected difference in reflectivity between the elastic true model and the acoustic inversion. The density is inside the differential operator in the variable density viscoacoustic wave equation, and it is difficult to calculate the density perturbation based on the framework of EWI. Thus, we add density 
to the inversion using a gradient-based method in the conventional FWI (Bai and Yingst, 2014; Luo and Wu, 2018). This process requires to calculate the background and adjoint wavefields, so it will increase the computational cost. Though FWI implementation for density will cause the nonlinear issue, it will not affect the velocity and $Q$ inversion. Because we do not expect to invert for a reasonable density model, but use the density inversion to handle the amplitude discrepancies between the acoustic modeled and elastic observed data (Guitton and Alkhalifah, 2017). Density inversion can be used to absorb noise-related to S-wave arrivals and near-offset reflections (Przebindowska et al., 2012; Guitton, 2014; Li et al., 2019). The density is inverted simultaneously with the velocity, and the initial density model used in the test is a constant scale of the initial velocity model, as shown in Figure 24. After ten outer iterations of updating velocity and density first, and $Q$ model second, the inversion results of the velocity and $Q$ are shown in Figures $25 \mathrm{a}$ and $25 \mathrm{~b}$, respectively. In the velocity inversion, we keep the water layer and a thin deep layer fixed. We observe that the main structures of the Overthrust velocity is reasonably recovered, and we successfully capture the high intrinsic attenuation area in the shallow part. As for the density model, it is sensitive to small scattering angles. As a result, some high-wavenumber components are absorbed by the density model, as shown in Figure 26. Using the same inversion setup and strategy, we repeat this experiment with viscoacoustic FWI with variable density, and the inverted velocity and $Q$ models are shown in Figures 27a and 27b, respectively. Clearly, they are not comparable to what we obtained using sequential viscoacoustic EWI. We perform the density inversion simultaneously with the velocity inversion and show the inversion result in Figure 28. Finally, we show in Figure 29 vertical velocity profiles at $7.5 \mathrm{~km}$, and we find that the inverted velocity using sequential viscoacoustic EWI agrees generally well with the true velocity. 


\section{DISCUSSIONS}

We use an efficient wavefield inversion (EWI) to perform a multiparameter waveform inversion for viscoacoustic media. EWI uses the modified source $f_{e}$ to absorb the parameter perturbation, and it starts with the true source function in the beginning of the inversion process. The reconstructed wavefield initially contains only single scattering components. After updating the $f_{e}$ with the reconstructed wavefield, secondary sources (media parameter perturbations) are added to $f_{e}$. Then, the inner iterations transfer the scattering components to the wavefield. The more inner iterations, the higher the order of scattering information is included in the wavefield. Scattering energy higher than the second order is relatively weak to contribute to the parameter update (Alkhalifah and Wu, 2016; Song et al., 2019b), so we use two inner iterations in our implementation.

The weighting factor $\alpha^{2}$ in the objective function is important for avoiding cycleskipping. A large $\alpha^{2}$ means more focus on the wave equation. In this case, the less data are used to construct the wavefield. A small $\alpha^{2}$ means weight to the data fitting component of the inversion. As a result, the cycle-skipping problem can be somewhat mitigated (Alkhalifah, 2020). It is always a good idea to do some trial and error tests to find an appropriate $\alpha^{2}$. A quick way to find a proper $\alpha^{2}$ is to scale the highest eigenvalue of the augmented linear system (van Leeuwen and Herrmann, 2015). The weight $\alpha^{2}$ can also be automatically selected by using a multiplicative cost function (da Silva and Yao, 2017). Although EWI is reliable and efficient, it fails to reconstruct the wavefields and invert for a reasonable velocity when the required velocity perturbation is large, no matter how much we control the weighting factor (Song and Alkhalifah, 2020b).

The velocity and intrinsic attenuation parameters share similar radiation patterns with 
different magnitude sensitivities to the data. A simultaneous inversion for the velocity and the $Q$ may cause an inaccurate update of the latter due to the large error in the former. We recommend to use a sequential scheme to invert for the velocity and the intrinsic attenuation parameters, though it may add some extra cost. This is affordable, because the EWI implementation does not require the calculation of the adjoint wavefield. The velocity perturbation including the multiscattering information is calculated directly without the requirement for gradient smoothing or optimization. By doing this, the velocity update leakage will not affect the $Q$ inversion result. As we always update the velocity model first, the error in the $Q$ model will affect the velocity inversion accuracy at the early stages. However, as the $Q$ model matures through the outer iterations, the velocity error reduces. As a result, the $Q$ model inversion accuracy also improves with outer iterations.

The inverted $Q$ model tends to have many oscillatory artifacts, and we use an iterative TV denoising method to remove all these artifacts in each outer iteration. In the $Q$ inversion, a smoothing operator or regularization are recommended (Malinowski et al., 2011; Kamei and Pratt, 2013). This process aims to remove the high-wavenumber velocity update leakage, which also results in the lack of high wavenumbers in the inverted models of $Q$. Reflections are sensitive to the high-wavenumber components of the velocity, not to the $Q$ model. So it is difficult to retrieve high-wavenumber updates in the intrinsic attenuation. When the intrinsic attenuation only exists locally in a high- $Q$ background, it will not affect the velocity inversion dramatically. On the other hand, an accurate description of the $Q$ model will improve the velocity inversion result.

The proposed method is implemented in the frequency domain, and the inner iterations are only efficient with the help of an LU decomposition. However, LU decomposition will be extremely expensive for 3D applications. A dual formulation for time-domain WRI is 
introduced (Rizzuti et al., 2019) which makes large-scale seismic problems feasible. However, it still requires twice as much the cost needed for the conventional FWI. An efficient timedomain implementation for the wavefield-reconstruction based method still needs further research. Though we do not use a free-surface condition in our synthetic examples, it will not be a problem using the free-surface condition, as shown in related work (Aghamiry et al., 2019). We recommend to use the free-surface condition in the real data application to reduce the surface-related multiple influences.

\section{CONCLUSIONS}

We use an efficient wavefield inversion to implement a multiparameter estimation in viscoacoustic media. We invert for the velocity and the intrinsic attenuation parameter in a sequential matter to reduce the trade-off noise. As the parameter perturbations are calculated using direct division, the computational cost is affordable. A test on a model with distinct Gaussian anomalies for the velocity and the intrinsic attenuation parameter shows that a sequential EWI can mitigate the trade-off problem effectively. Applications on synthetic data generated from an intrinsic attenuation Marmousi and an intrinsic attenuation Overthrust models show that EWI can reasonably recover the viscoacoustic parameters.

\section{ACKNOWLEDGMENTS}

We thank KAUST for its support and the SWAG group for the collaborative environment. We thank the editor Colin Farquharson, the associate editor Rie Nakata (Kamei), and three anonymous reviewers for their important suggestions and comments. We also thank the Center for Subsurface Imaging and Modeling (CSIM) group releasing the viscoacoustic Marmousi and Overthrust models. 


\section{REFERENCES}

Aghamiry, H., A. Gholami, and S. Operto, 2019, Joint estimation of velocity and attenuation by frequency-domain tv-regularized wavefield reconstruction inversion: 81st EAGE Conference and Exhibition 2019 Workshop Programme, European Association of Geoscientists \& Engineers, 1-5.

Aki, K., and P. G. Richards, 2002, Quantitative seismology.

Alkhalifah, T., 2020, Linear wavefield optimization using a modified source: Communications in computational physics, 28, 276-296.

Alkhalifah, T., and C. Song, 2019, An efficient wavefield inversion: Using a modified source function in the wave equation: Geophysics, 84, no.6, R921-R934.

Alkhalifah, T., and Z. Wu, 2016, Multiscattering inversion for low-model wavenumbers: Geophysics, 81, no.6, R417-R428.

Amestoy, P., R. Brossier, A. Buttari, J.-Y. LExcellent, T. Mary, L. Métivier, A. Miniussi, and S. Operto, 2016, Fast 3D frequency-domain full-waveform inversion with a parallel block low-rank multifrontal direct solver: Application to OBC data from the North Sea 3D FD FWI with BLR direct solver: Geophysics, 81, no.6, R363-R383.

Bai, J., and D. Yingst, 2014, Simultaneous inversion of velocity and density in time-domain full waveform inversion, in SEG Technical Program Expanded Abstracts 2014: Society of Exploration Geophysicists, 922-927.

Blanch, J. O., J. O. Robertsson, and W. W. Symes, 1995, Modeling of a constant Q: Methodology and algorithm for an efficient and optimally inexpensive viscoelastic technique: Geophysics, 60, 176-184.

da Silva, N. V., and G. Yao, 2017, Wavefield reconstruction inversion with a multiplicative cost function: Inverse Problems, 34, 015004. 
da Silva, N. V., G. Yao, and M. Warner, 2019, Semiglobal viscoacoustic full-waveform inversion: Geophysics, 84, no.2, R271-R293.

Dutta, G., and G. T. Schuster, 2014, Attenuation compensation for least-squares reverse time migration using the viscoacoustic-wave equation: Geophysics, 79, no.6, S251-S262.

Gao, F., and Y. Wang, 2016, Simultaneous inversion for velocity and attenuation by waveform tomography: Journal of Applied Geophysics, 131, 103-108.

Guitton, A., 2014, On the velocity-density ambiguity in acoustic full-waveform inversion: 76th EAGE Conference and Exhibition 2014, European Association of Geoscientists \& Engineers, We E106 03.

Guitton, A., and T. Alkhalifah, 2017, A parameterization study for elastic VTI fullwaveform inversion of hydrophone components: Synthetic and North Sea field data examples: Geophysics, 82, no.6, R299-R308.

Hu, W., J. Liu, L. Bear, and C. Marcinkovich, 2011, A robust and accurate seismic attenuation tomography algorithm, in SEG Technical Program Expanded Abstracts 2011: Society of Exploration Geophysicists, 2727-2731.

Kalita, M., V. Kazei, Y. Choi, and T. Alkhalifah, 2019, Regularized full-waveform inversion with automated salt flooding: Geophysics, 84, no.4, R569-R582.

Kamei, R., and R. Pratt, 2013, Inversion strategies for visco-acoustic waveform inversion: Geophysical Journal International, 194, 859-884.

Kamei, R., and R. G. Pratt, 2008, Waveform tomography strategies for imaging attenuation structure with cross-hole data: 70th EAGE Conference and Exhibition incorporating SPE EUROPEC 2008, European Association of Geoscientists \& Engineers, F019.

Li, Y., Q. Guo, Z. Li, and T. Alkhalifah, 2019, Elastic reflection waveform inversion with variable density: Geophysics, 84, no.4, R553-R567. 
Luo, J., and R.-S. Wu, 2018, Velocity and density reconstruction based on scattering angle separation: Pure and Applied Geophysics, 175, 4371-4387.

Malinowski, M., S. Operto, and A. Ribodetti, 2011, High-resolution seismic attenuation imaging from wide-aperture onshore data by visco-acoustic frequency-domain fullwaveform inversion: Geophysical Journal International, 186, 1179-1204.

Métivier, L., R. Brossier, J. Virieux, and S. Operto, 2013, Full waveform inversion and the truncated newton method: SIAM Journal on Scientific Computing, 35, no.2, B401-B437.

Plessix, R.-E., 2006, A review of the adjoint-state method for computing the gradient of a functional with geophysical applications: Geophysical Journal International, 167, 495503.

Pratt, R. G., 1999, Seismic waveform inversion in the frequency domain, part 1: Theory and verification in a physical scale model: Geophysics, 64, 888-901.

Pratt, R. G., C. Shin, and G. Hick, 1998, Gauss-Newton and full Newton methods in frequency-space seismic waveform inversion: Geophysical journal international, 133, 341362.

Przebindowska, A., A. Kurzmann, D. Köhn, and T. Bohlen, 2012, The role of density in acoustic full waveform inversion of marine reflection seismics: 74th EAGE Conference and Exhibition incorporating EUROPEC 2012, European Association of Geoscientists \& Engineers, cp-293.

Quan, Y., and J. M. Harris, 1997, Seismic attenuation tomography using the frequency shift method: Geophysics, 62, 895-905.

Rizzuti, G., M. Louboutin, R. Wang, E. Daskalakis, and F. Herrmann, 2019, A dual formulation for time-domain wavefield reconstruction inversion, in SEG Technical Program Expanded Abstracts 2019: Society of Exploration Geophysicists, 1480-1485. 
Robertsson, J. O., J. O. Blanch, and W. W. Symes, 1994, Viscoelastic finite-difference modeling: Geophysics, 59, 1444-1456.

Rudin, L. I., S. Osher, and E. Fatemi, 1992, Nonlinear total variation based noise removal algorithms: Physica D: nonlinear phenomena, 60, 259-268.

Song, C., and T. Alkhalifah, 2020a, An efficient wavefield inversion for transversely isotropic media with a vertical axis of symmetry: Geophysics, 85, no.3, R195-R206.

$\longrightarrow, 2020 \mathrm{~b}$, Efficient wavefield inversion with outer iterations and total-variation constrain: IEEE Transactions on Geoscience and Remote Sensing, 1-11.

Song, C., T. Alkhalifah, G. Wang, and Q. Yang, 2019a, An efficient wavefield inversion for isotropic elastic media, in SEG Technical Program Expanded Abstracts 2019: Society of Exploration Geophysicists, 1575-1579.

Song, C., Z. Wu, and T. Alkhalifah, 2019b, Passive seismic event estimation using multiscattering waveform inversion: Geophysics, 84, no.3, KS59-KS69.

Tarantola, A., 1984, Inversion of seismic reflection data in the acoustic approximation: Geophysics, 49, 1259-1266.

— 1994, On: "Sensitivity of reflected and transmitted seismic data," by Rune Mittet and Tom Houlder (November 1993 Geophysics, 58, 1621-1628): Geophysics, 59, 14571458.

Toksöz, M., and D. Johnston, 1981, Geophysics reprint series, no. 2: Seismic wave attenuation: Society of exploration geophysicists.

Van Leeuwen, T., and F. J. Herrmann, 2013, Mitigating local minima in full-waveform inversion by expanding the search space: Geophysical Journal International, 195, 661667. 
verse Problems, 32, 015007.

Vogel, C. R., and M. E. Oman, 1996, Iterative methods for total variation denoising: SIAM Journal on Scientific Computing, 17, 227-238.

Wang, S., M. V. de Hoop, and J. Xia, 2011, On 3D modeling of seismic wave propagation via a structured parallel multifrontal direct Helmholtz solver: Geophysical Prospecting, 59, 857-873.

Xue, Z., J. Sun, S. Fomel, and T. Zhu, 2017, Accelerating full-waveform inversion with attenuation compensation: Geophysics, 83, no.1, A13-A20.

Yang, P., R. Brossier, L. Métivier, J. Virieux, and W. Zhou, 2018, A time-domain preconditioned truncated Newton approach to visco-acoustic multiparameter full waveform inversion: SIAM Journal on Scientific Computing, 40, B1101-B1130.

Zhu, T., J. M. Harris, and B. Biondi, 2014, Q-compensated reverse-time migration: Geophysics, 79, no.3, S77-S87. 


\section{LIST OF FIGURES}

1 The true Gaussian anomaly velocity model (a), and the Gaussian anomaly $Q$ model

(b) with the source geometry (symbols $*$ denote source locations).

2 The inverted velocity models using joint (a), and sequential (b) viscoacoustic EWI.

3 (a) The velocity difference between Figures $1 \mathrm{a}$ and $2 \mathrm{a}$, and (b) difference between

Figures $1 \mathrm{a}$ and $2 \mathrm{~b}$.

4 The inverted $Q$ models using joint (a), and sequential (b) viscoacoustic EWI.

5 The normalized data misfit per iteration of joint (blue curve) and sequential (red curve) viscoacoustic EWI.

6 The true (a), and initial (b) Marmousi velocity model with the source geometry (symbols $*$ denote source locations).

$7 \quad$ The true Marmousi $Q$ model.

8 The inverted velocity models using acoustic FWI (a), and acoustic EWI (b). Black circle indicates the poorly inverted area using acoustic EWI.

9 The inverted velocity models with the fixed background $Q$ (a), and with $Q$ inversion included (b) using the sequential viscoacoustic EWI. Black arrows indicate the velocity inversion improvements using the sequential viscoacoustic EWI.

10 The inverted $Q$ model using sequential viscoacoustic EWI.

11 The vertical velocity profiles at $3.75 \mathrm{~km}$ (a), and at $8.25 \mathrm{~km}$ (b) (black solid curve: true velocity, yellow solid curve: initial velocity, blue dashed curve: inverted velocity with background $Q$ model, red dotted curve: inverted velocity using the inverted $Q$ model).

12 QLSRTM image corresponding to the initial velocity and the background $Q$ (a), the acoustic inverted velocity and the background $Q(\mathrm{~b})$, and the viscoacoustic EWI inverted velocity and the inverted $Q$ (c). Red arrows indicate the image improvements. 
13 The inverted velocity models using sequential viscoacoustic EWI (a), sequential viscoacoustic FWI (b), and joint viscoacoustic EWI (c) schemes with TV denoising applied to the inverted $Q$ model. Black arrows indicate the velocity inversion improvements.

14 The inverted $Q$ models using sequential viscoacoustic EWI (a), sequential viscoacoustic FWI (b), and joint viscoacoustic EWI (c) schemes with TV denoising.

15 The percent RMS error of the inverted velocity models per iteration (green curve: acoustic FWI; black curve: viscoacoustic FWI; purple curve: acoustic EWI; red curve: viscoacoustic EWI with background $Q$ model; cyan curve: joint viscoacoustic EWI; blue curve: sequential viscoacoustic EWI).

16 The percent RMS error of the inverted $Q$ models per iteration (blue curve: sequential viscoacoustic FWI; red curve: joint viscoacoustic EWI; black curve: sequential viscoacoustic EWI).

17 The true (a), and initial (b) Overthrust velocity model with the source geometry (symbols $*$ denote source locations).

18 The true Overthrust $Q$ model.

19 The inverted velocity models using sequential viscoacoustic EWI (a), and sequential viscoacoustic FWI (b).

20 The inverted $Q$ models using sequential viscoacoustic EWI (a), and sequential viscoacoustic FWI (b) with TV denoising.

21 The real (a), and imaginary (a) parts of the clean and noisy data for a source in the middle at $5 \mathrm{~Hz}$. (red curve: clean data; blue dashed curve: noisy data)

22 The sequential viscoacoustic EWI inverted velocity (a), and $Q$ (a) models using noisy data with SNR of $10 \mathrm{db}$.

23 One shot gather from the time-domain data generated by viscoacoustic (a), and 
viscoelastic (b) wave equations. Red circled areas indicate the S-wave artifacts.

24 The initial density model using in the inversion.

25 The sequential viscoacoustic EWI inverted velocity model (a), and $Q$ model (b) using time-domain viscoelastic data.

26 The inverted density model in viscoacoustic EWI.

27 The sequential viscoacoustic FWI inverted velocity model (a), and $Q$ model (b) using time-domain viscoelastic data.

28 The inverted density model in viscoacoustic FWI.

29 The vertical velocity profile at $7.5 \mathrm{~km}$ (black solid curve: true velocity, green solid curve: initial velocity, red dotted curve: sequential viscoacoustic FWI inverted velocity, blue dash-dotted curve: sequential viscoacoustic EWI inverted velocity). 


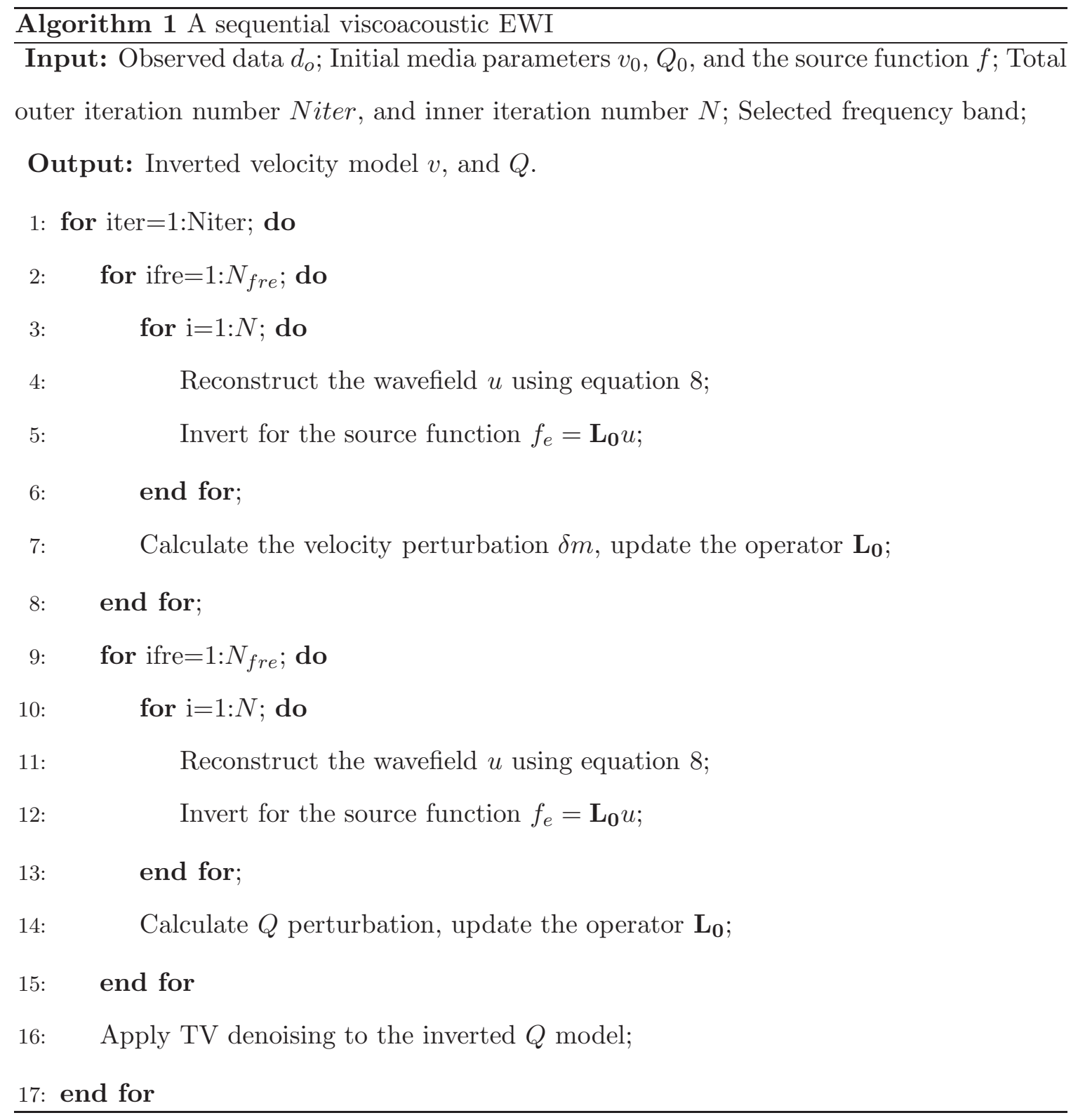



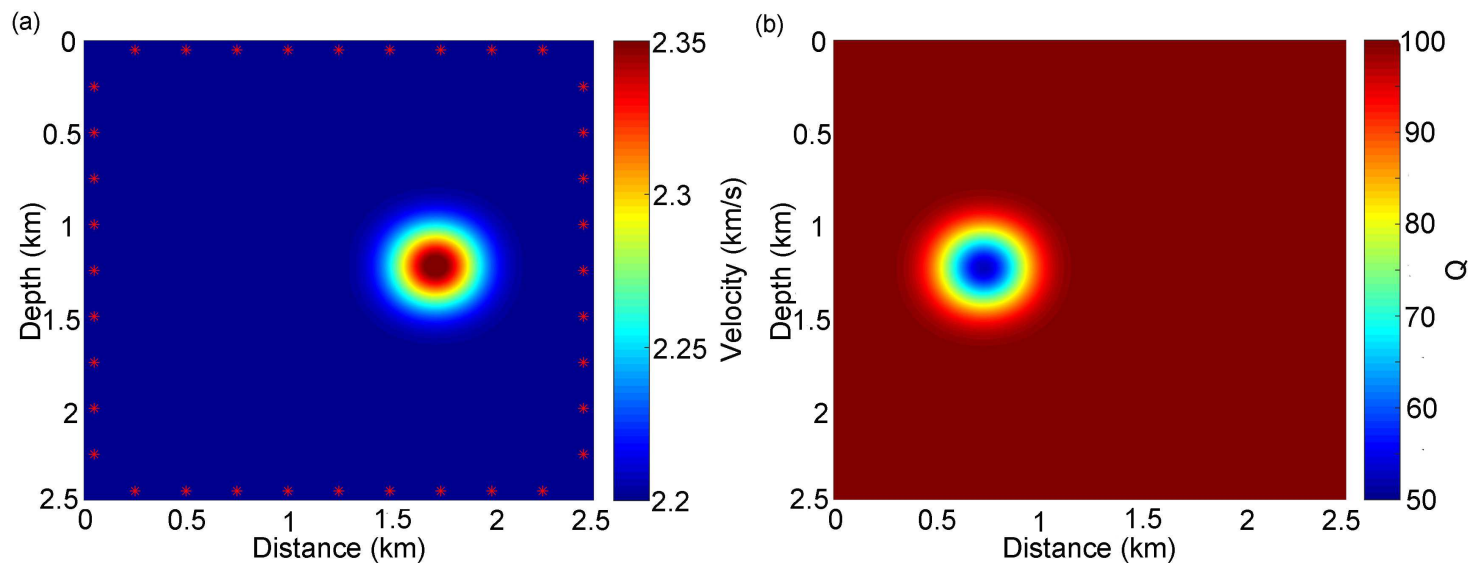

Figure 1: The true Gaussian anomaly velocity model (a), and the Gaussian anomaly $Q$ model (b) with the source geometry (symbols $*$ denote source locations). 

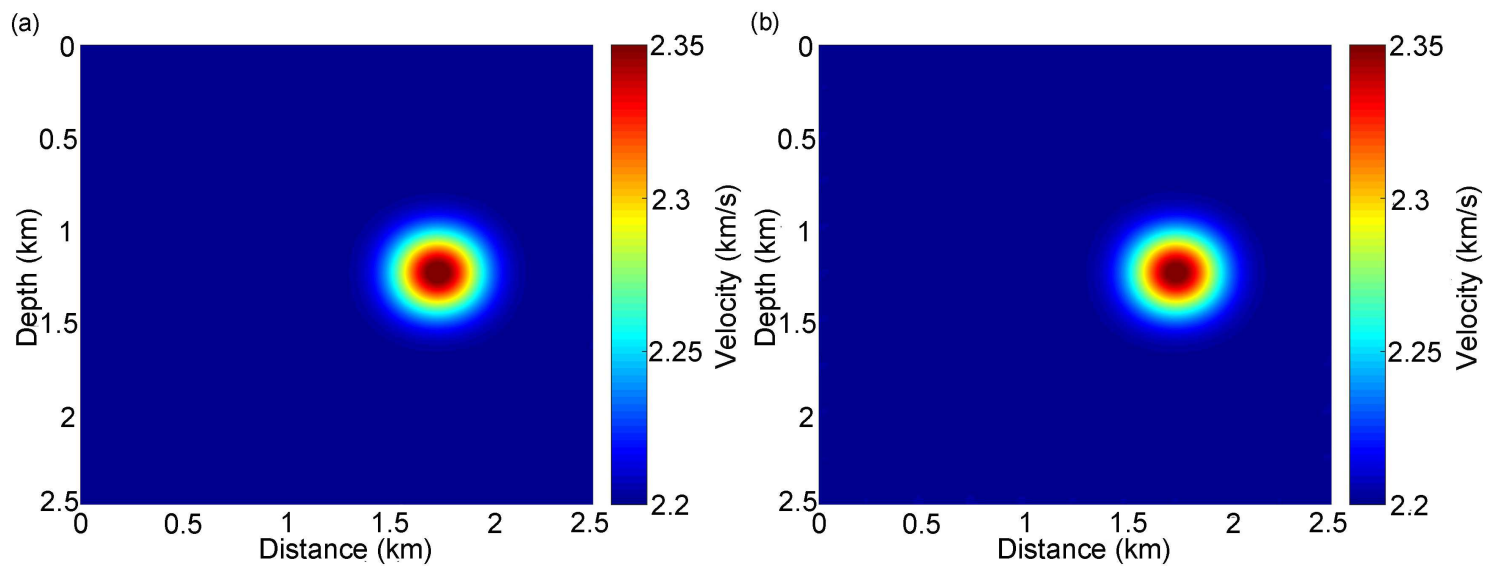

Figure 2: The inverted velocity models using joint (a), and sequential (b) viscoacoustic EWI. 


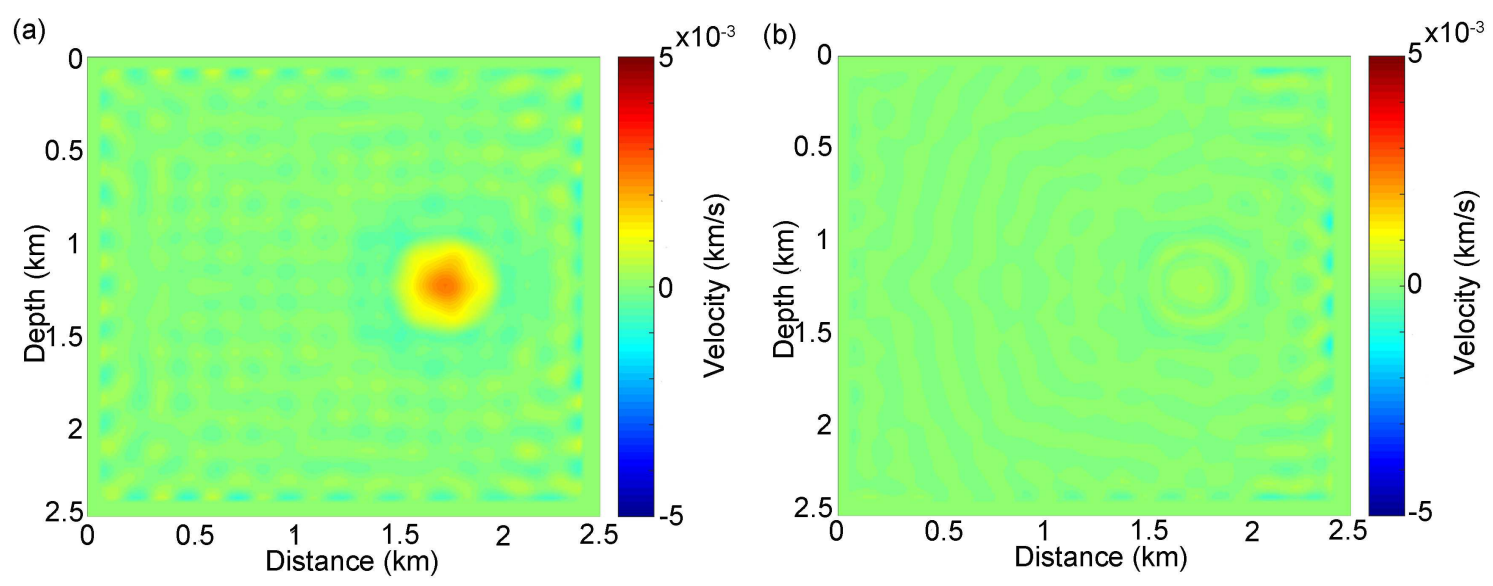

Figure 3: (a) The velocity difference between Figures 1a and 2a, and (b) difference between Figures $1 \mathrm{a}$ and $2 \mathrm{~b}$. 

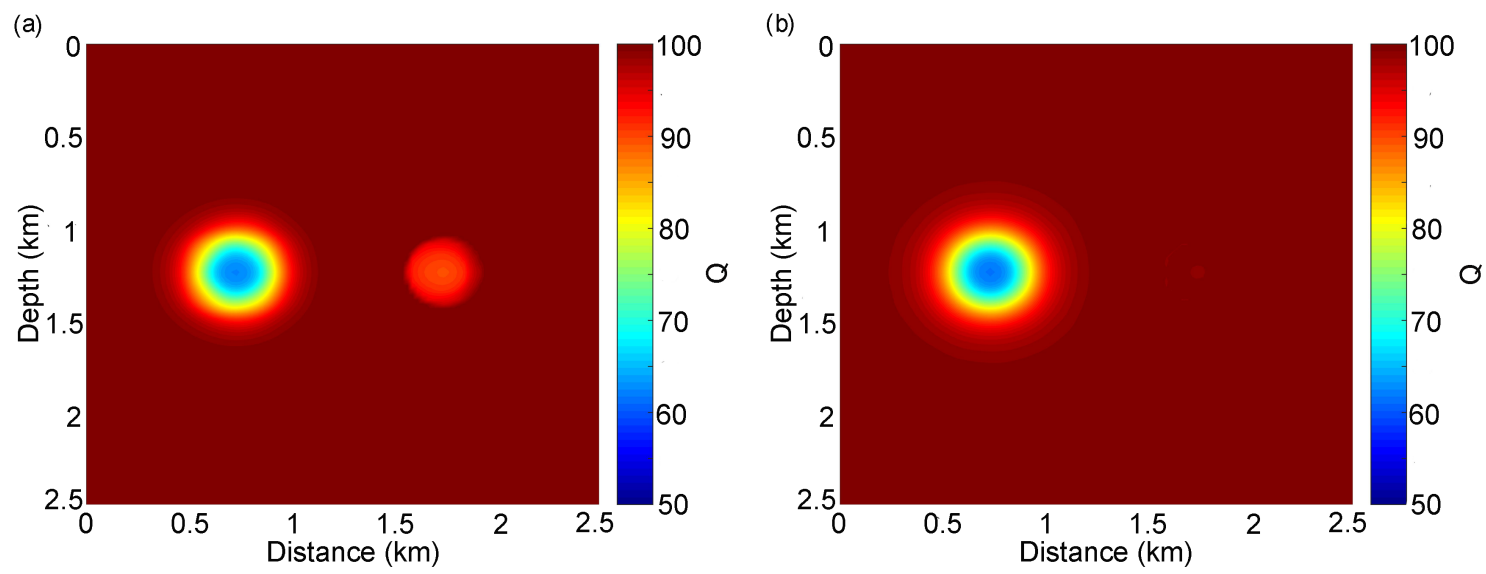

Figure 4: The inverted $Q$ models using joint (a), and sequential (b) viscoacoustic EWI. - 


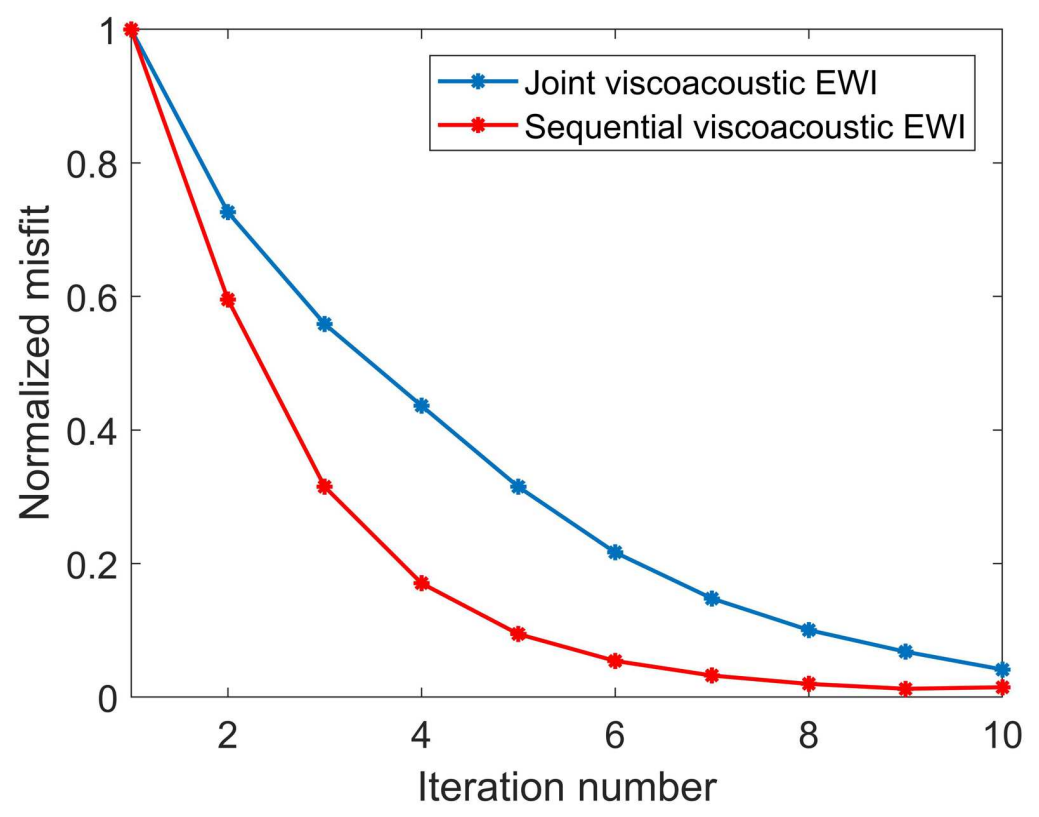

Figure 5: The normalized data misfit per iteration of joint (blue curve) and sequential (red curve) viscoacoustic EWI. 

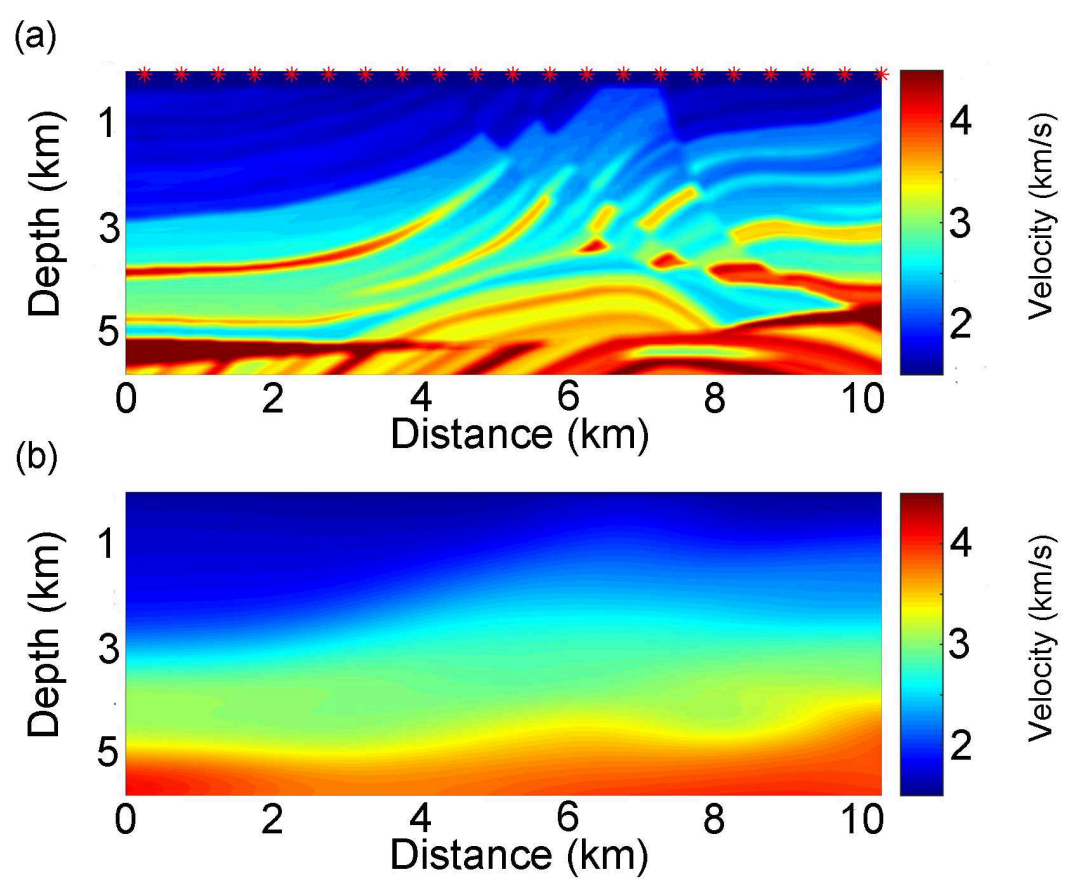

Figure 6: The true (a), and initial (b) Marmousi velocity model with the source geometry (symbols $*$ denote source locations). 


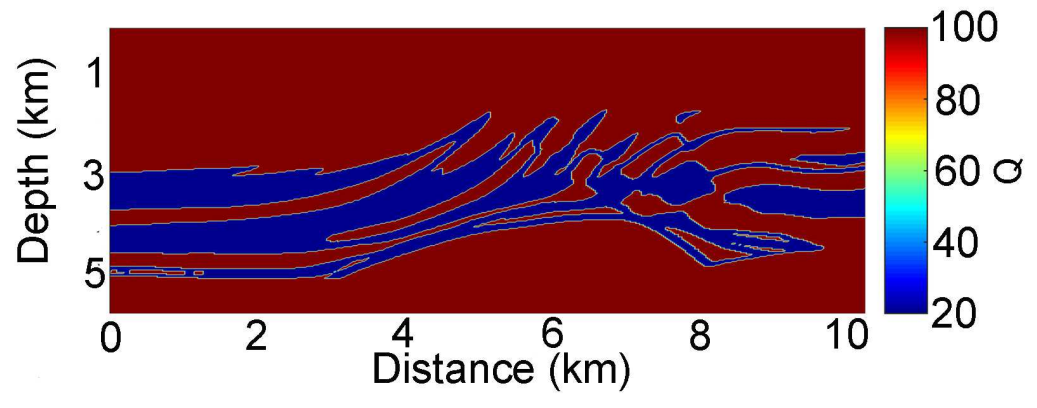

Figure 7: The true Marmousi $Q$ model. - 

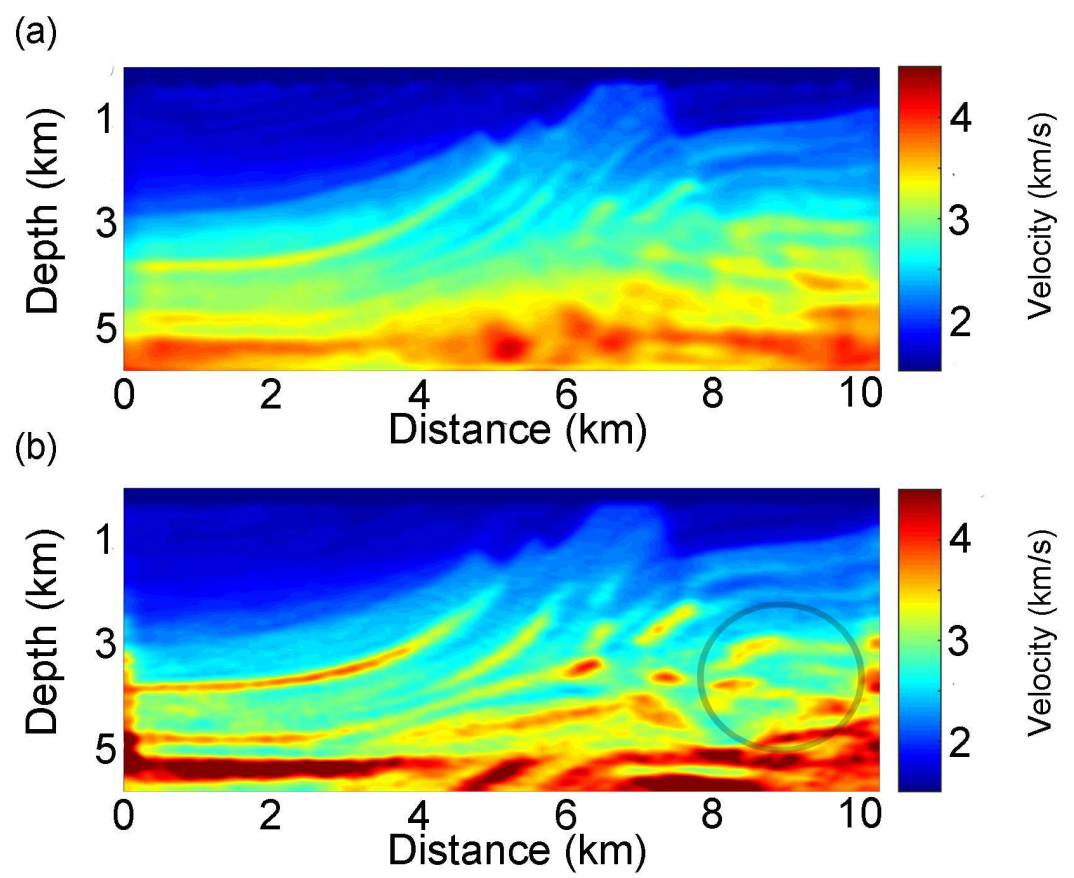

Figure 8: The inverted velocity models using acoustic FWI (a), and acoustic EWI (b). Black circle indicates the poorly inverted area using acoustic EWI. 


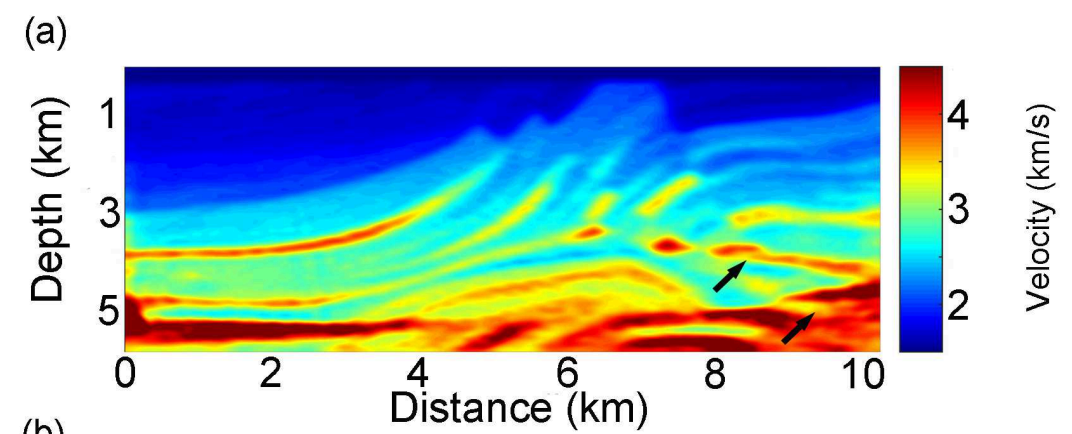

(b)

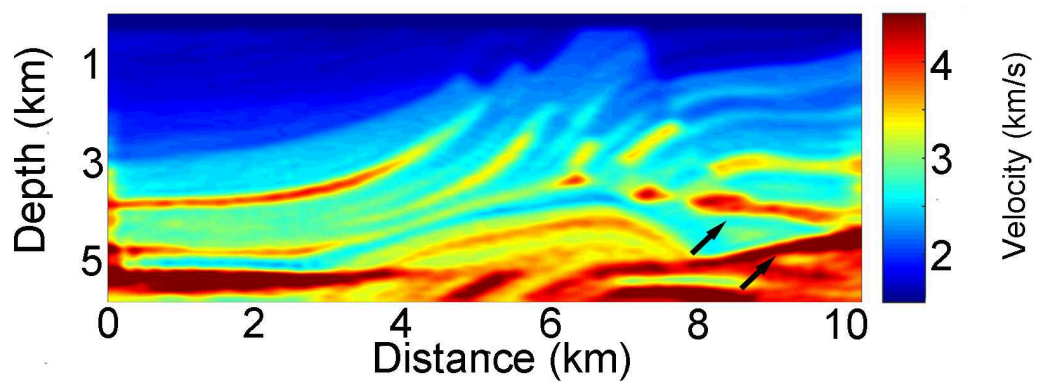

Figure 9: The inverted velocity models with the fixed background $Q$ (a), and with $Q$ inversion included (b) using the sequential viscoacoustic EWI. Black arrows indicate the velocity inversion improvements using the sequential viscoacoustic EWI. 


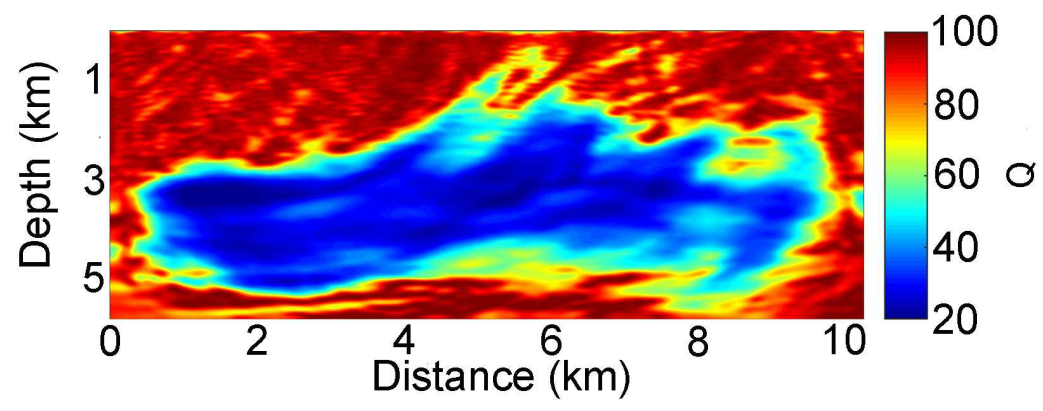

Figure 10: The inverted $Q$ model using sequential viscoacoustic EWI. - 

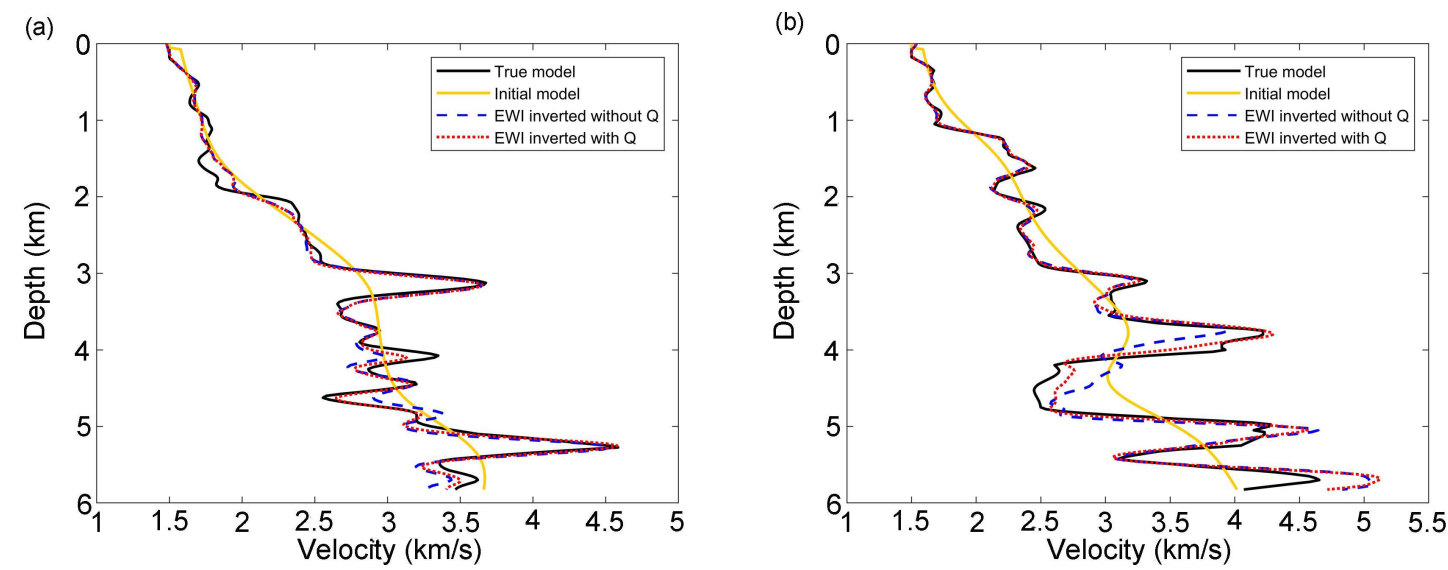

Figure 11: The vertical velocity profiles at $3.75 \mathrm{~km} \mathrm{(a),} \mathrm{and} \mathrm{at} 8.25 \mathrm{~km}$ (b) (black solid curve: true velocity, yellow solid curve: initial velocity, blue dashed curve: inverted velocity with background $Q$ model, red dotted curve: inverted velocity using the inverted $Q$ model). 

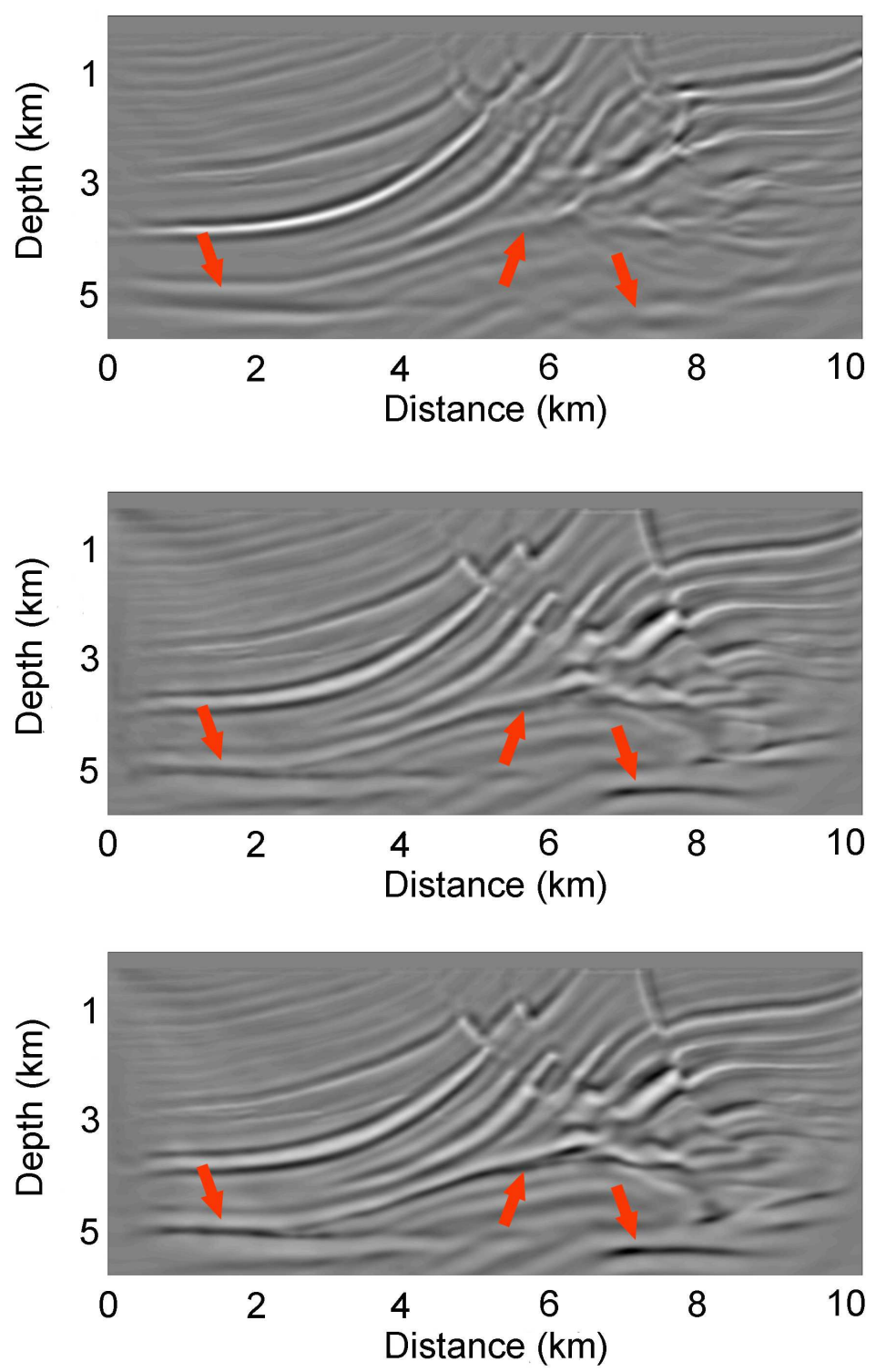

Figure 12: QLSRTM image corresponding to the initial velocity and the background $Q$ (a), the acoustic inverted velocity and the background $Q$ (b), and the viscoacoustic EWI inverted velocity and the inverted $Q$ (c). Red arrows indicate the image improvements. 

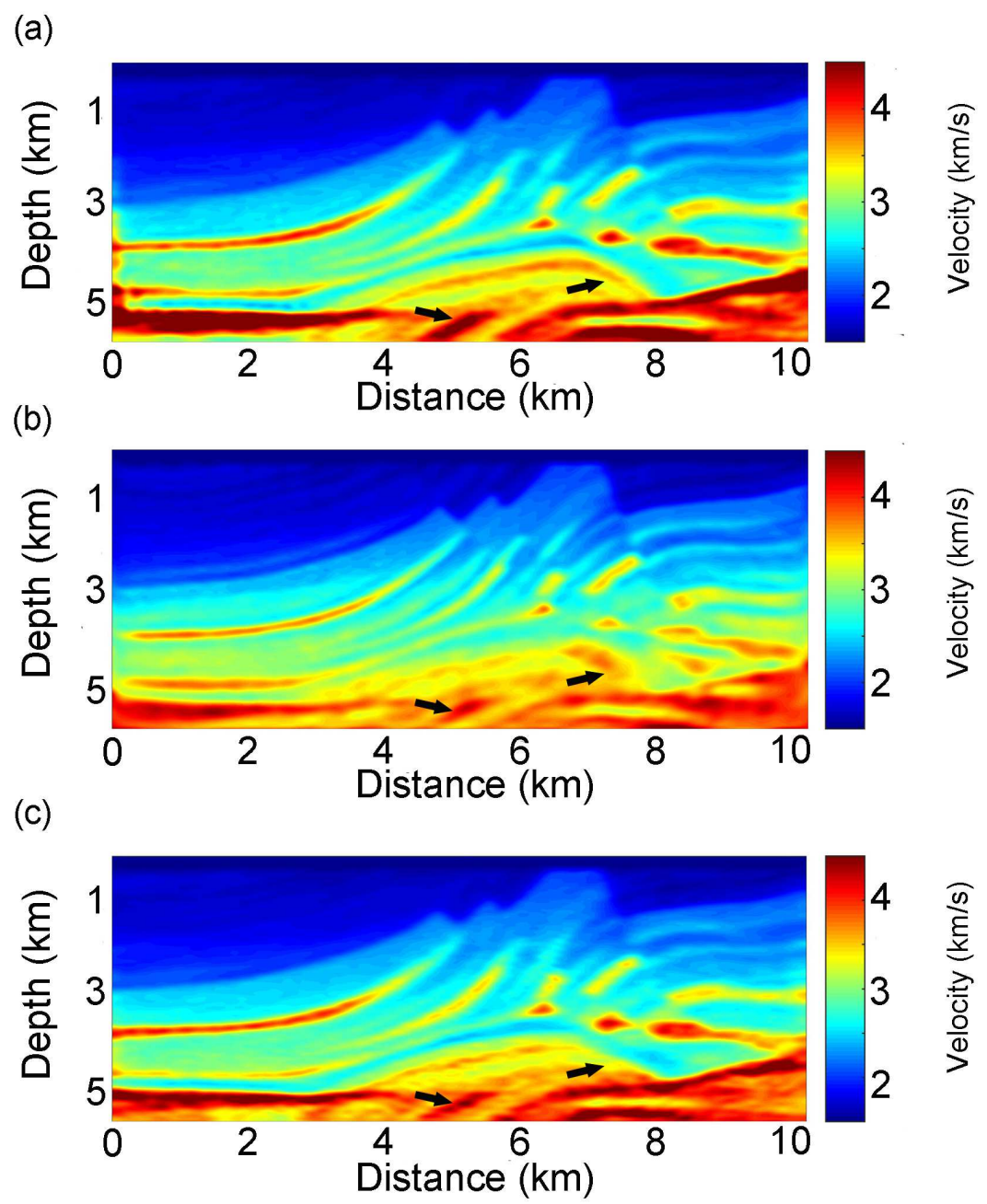

Figure 13: The inverted velocity models using sequential viscoacoustic EWI (a), sequential viscoacoustic FWI (b), and joint viscoacoustic EWI (c) schemes with TV denoising applied to the inverted $Q$ model. Black arrows indicate the velocity inversion improvements. 

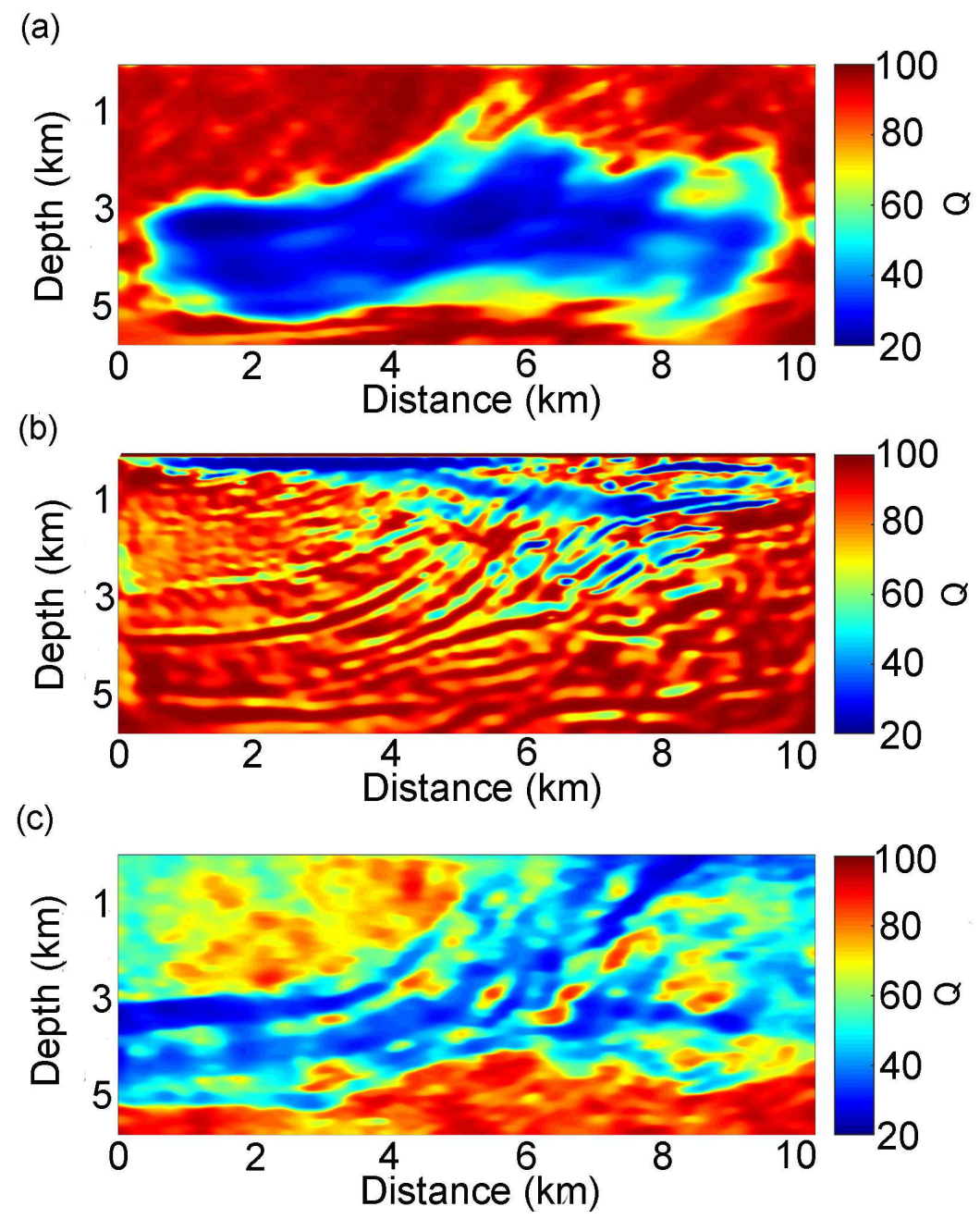

Figure 14: The inverted $Q$ models using sequential viscoacoustic EWI (a), sequential viscoacoustic FWI (b), and joint viscoacoustic EWI (c) schemes with TV denoising. 


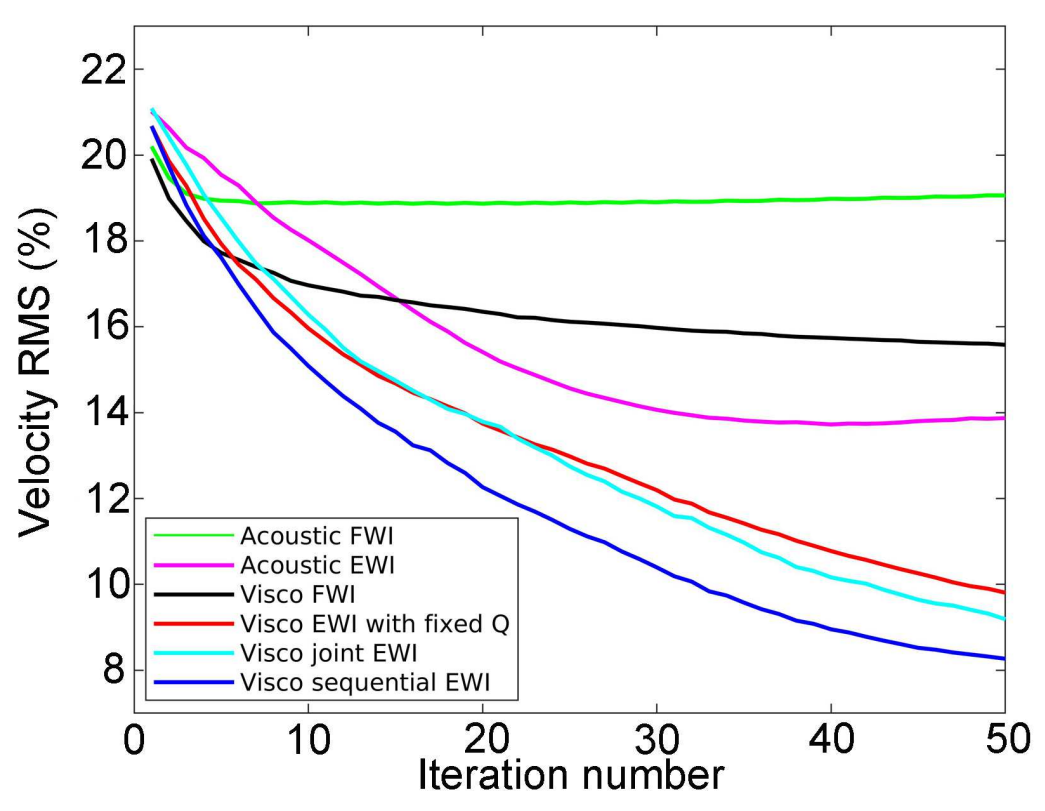

Figure 15: The percent RMS error of the inverted velocity models per iteration (green curve: acoustic FWI; black curve: viscoacoustic FWI; purple curve: acoustic EWI; red curve: viscoacoustic EWI with background $Q$ model; cyan curve: joint viscoacoustic EWI; blue curve: sequential viscoacoustic EWI). 


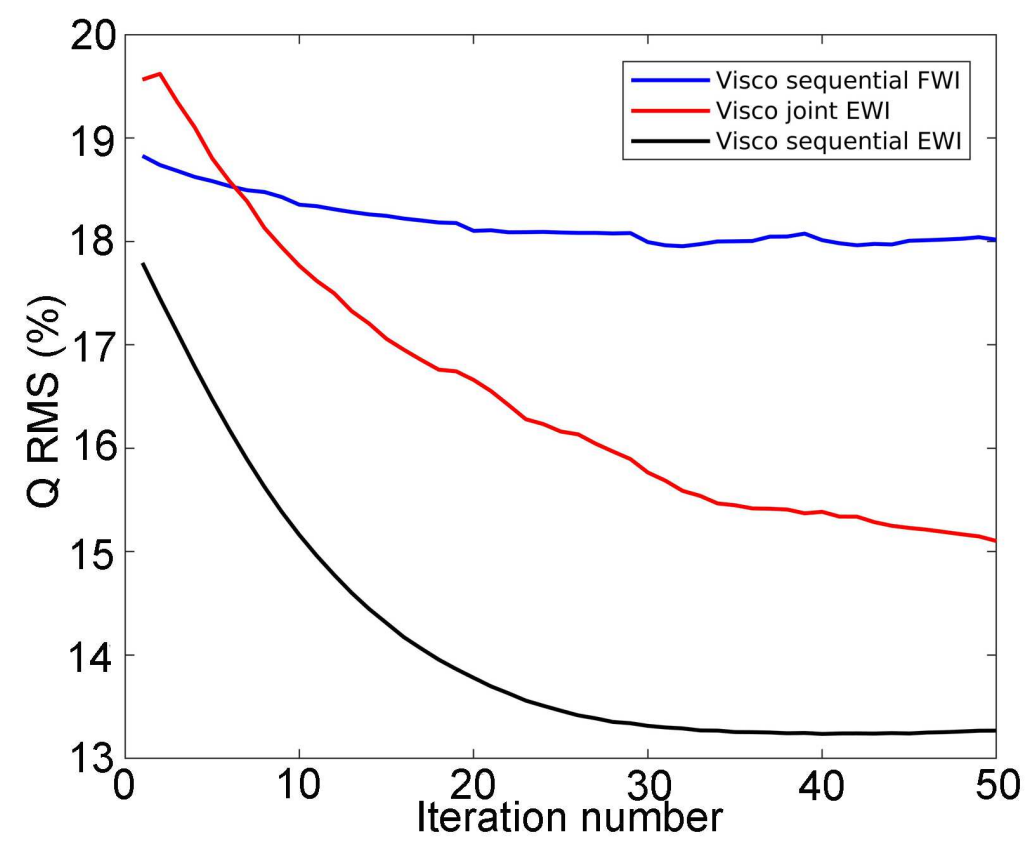

Figure 16: The percent RMS error of the inverted $Q$ models per iteration (blue curve: sequential viscoacoustic FWI; red curve: joint viscoacoustic EWI; black curve: sequential viscoacoustic EWI). 

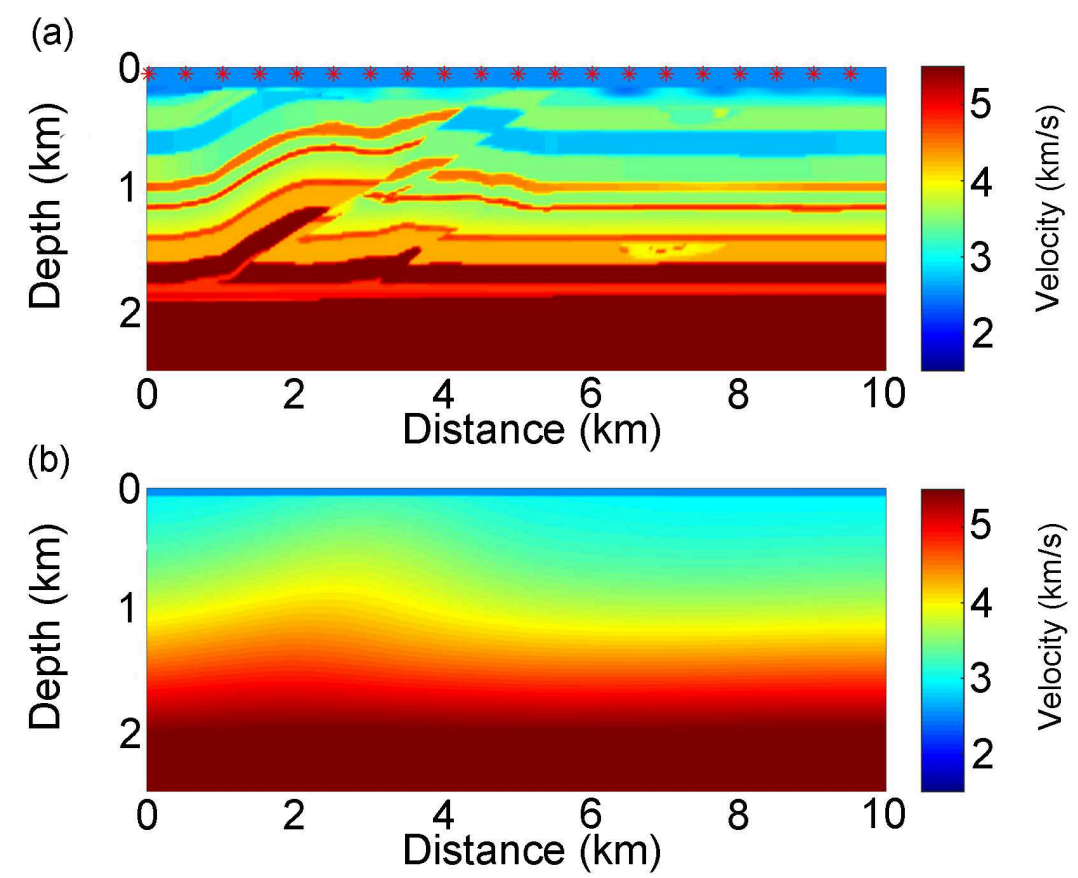

Figure 17: The true (a), and initial (b) Overthrust velocity model with the source geometry (symbols $*$ denote source locations). 


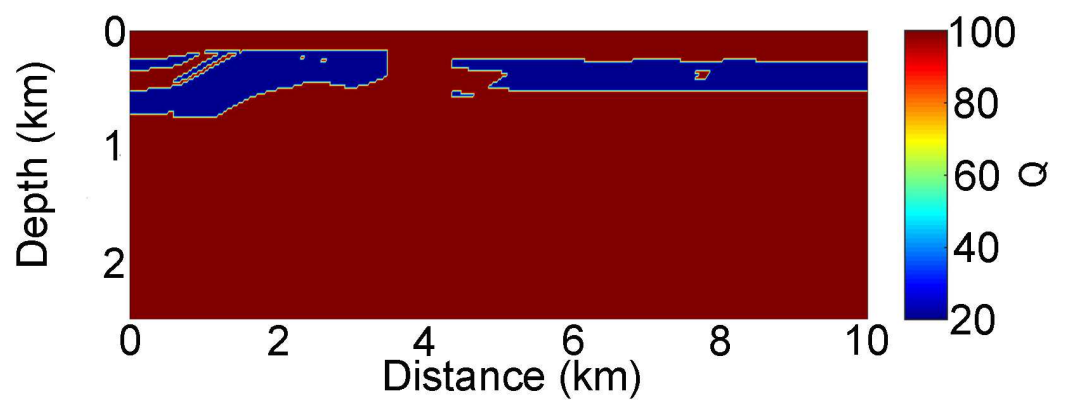

Figure 18: The true Overthrust $Q$ model. - 

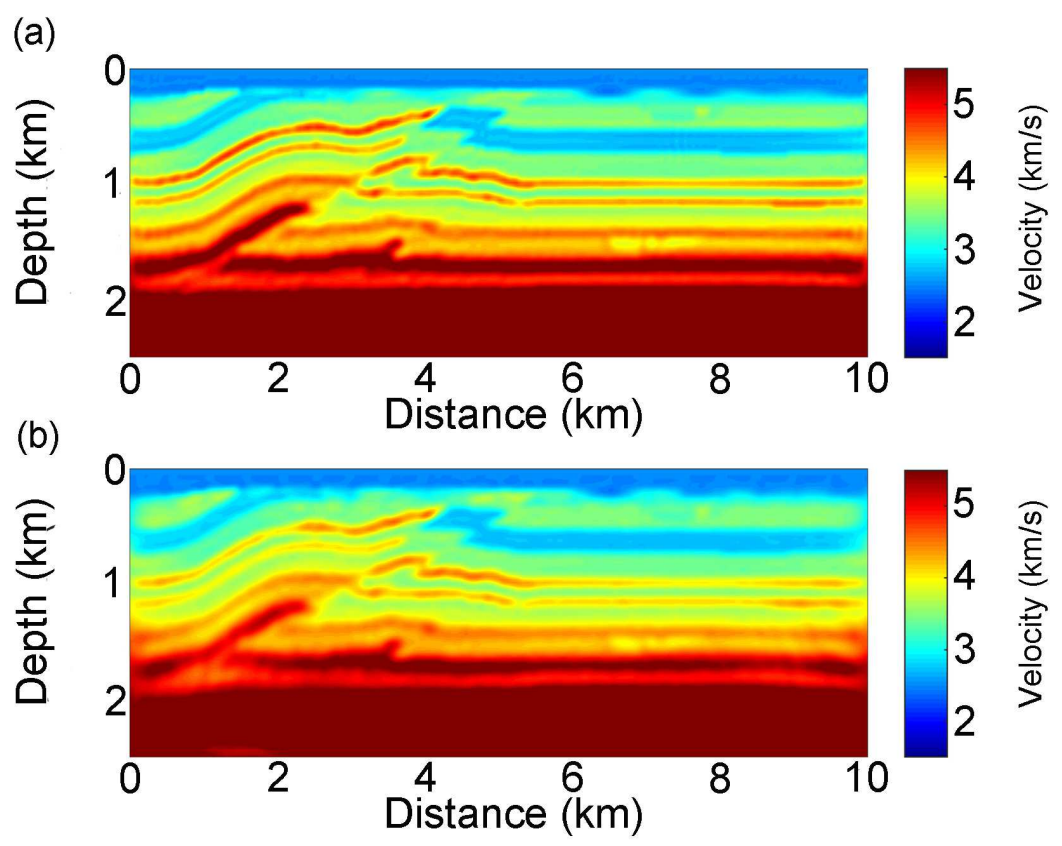

Figure 19: The inverted velocity models using sequential viscoacoustic EWI (a), and sequential viscoacoustic FWI (b). 

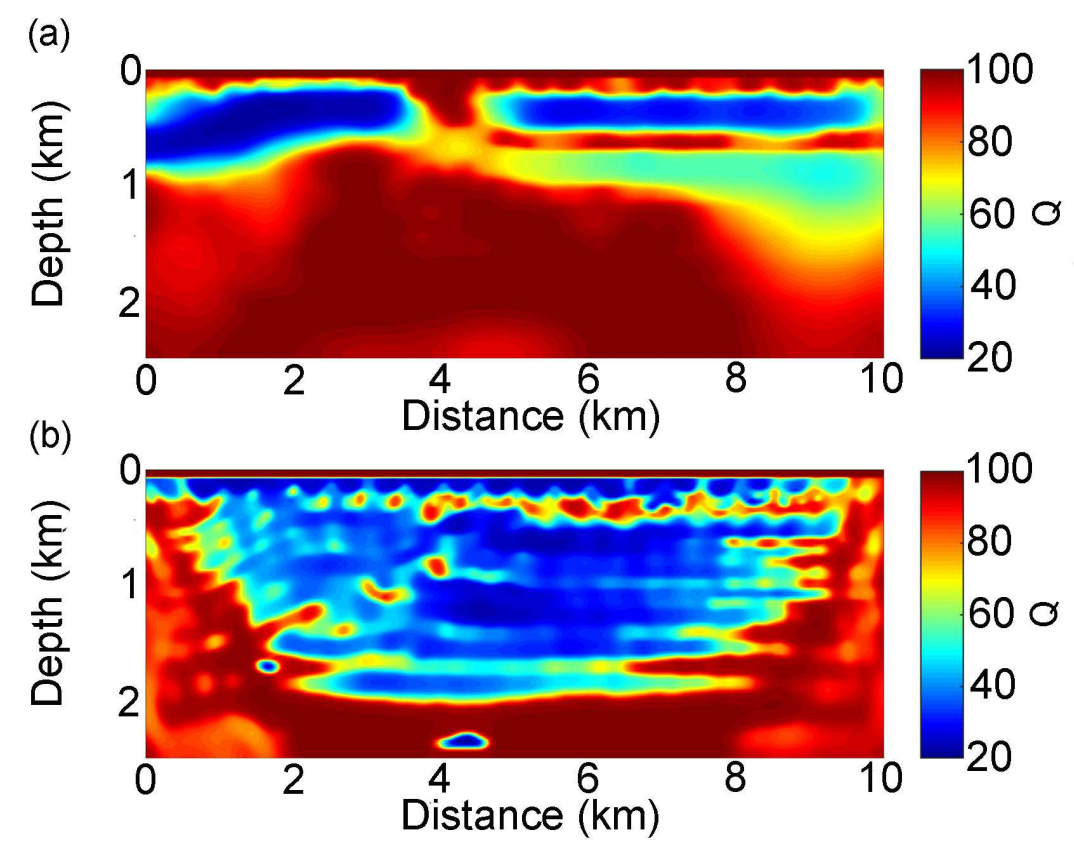

Figure 20: The inverted $Q$ models using sequential viscoacoustic EWI (a), and sequential viscoacoustic FWI (b) with TV denoising. 

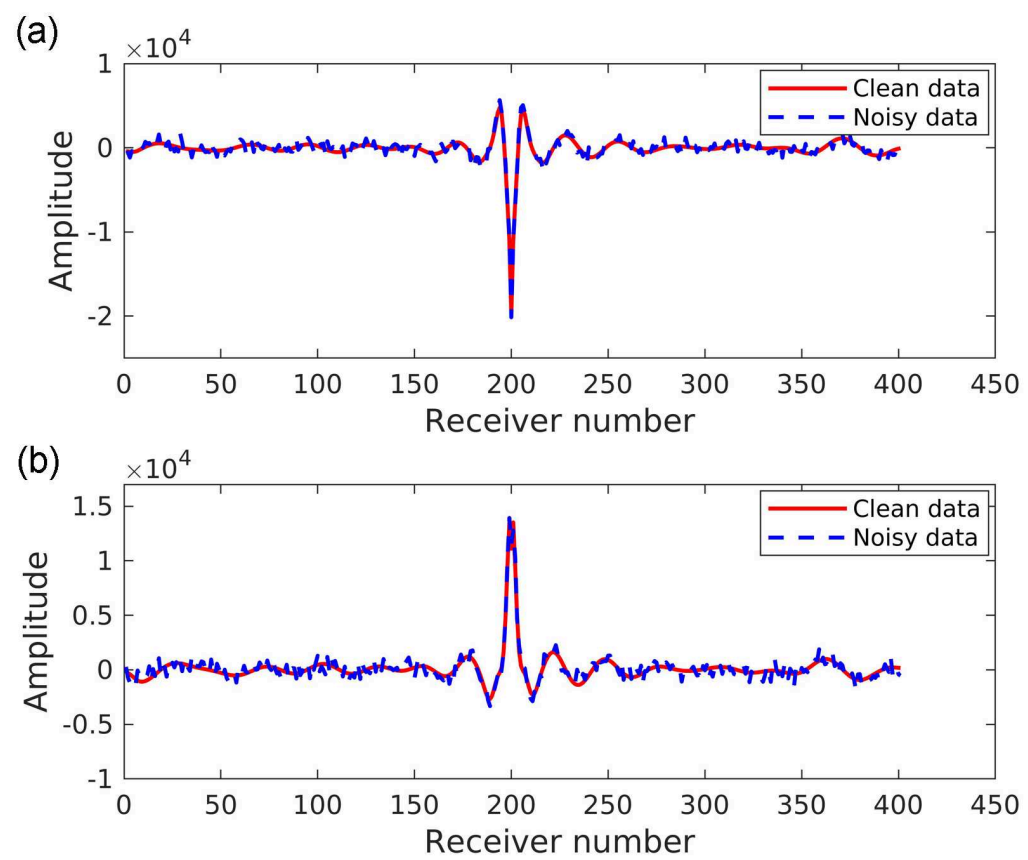

Figure 21: The real (a), and imaginary (a) parts of the clean and noisy data for a source in the middle at $5 \mathrm{~Hz}$. (red curve: clean data; blue dashed curve: noisy data) 

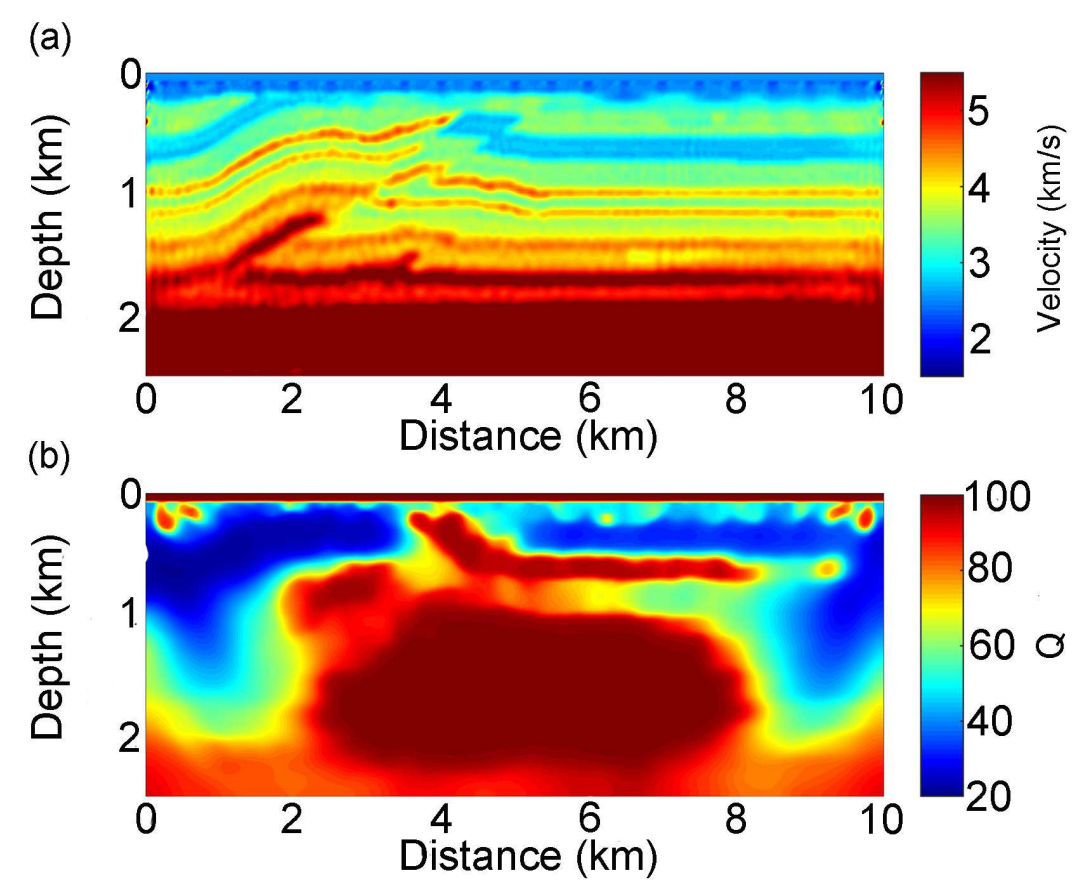

Figure 22: The sequential viscoacoustic EWI inverted velocity (a), and $Q$ (a) models using noisy data with SNR of $10 \mathrm{db}$. 
(a)

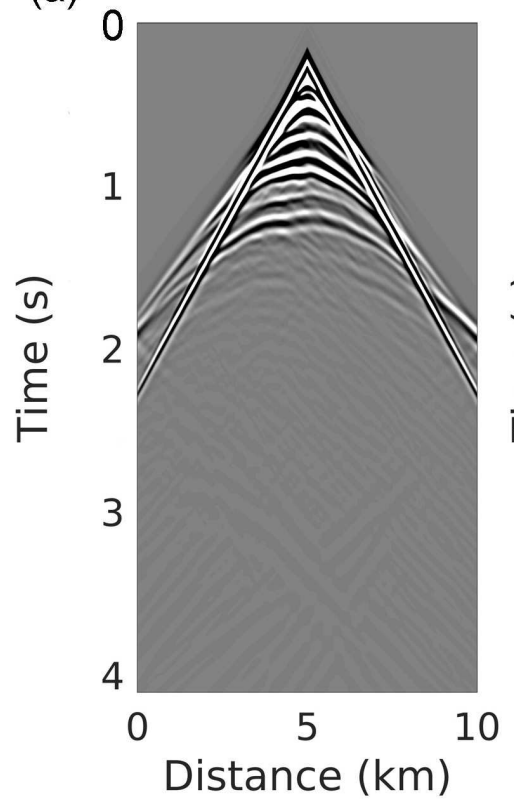

(b)

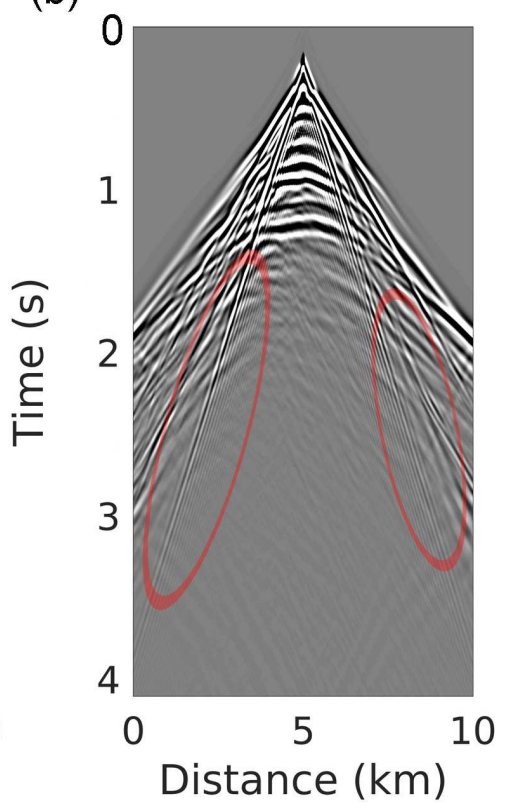

Figure 23: One shot gather from the time-domain data generated by viscoacoustic (a), and viscoelastic (b) wave equations. Red circled areas indicate the S-wave artifacts. 


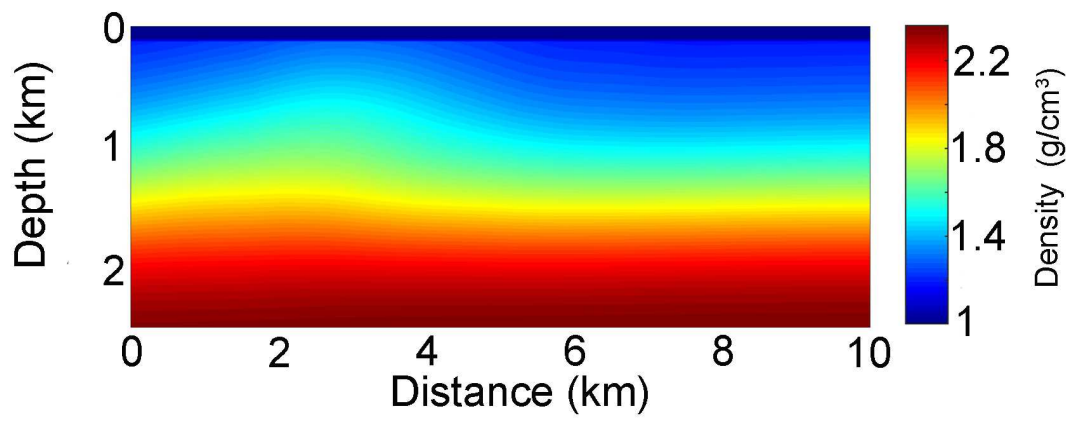

Figure 24: The initial density model using in the inversion. - 

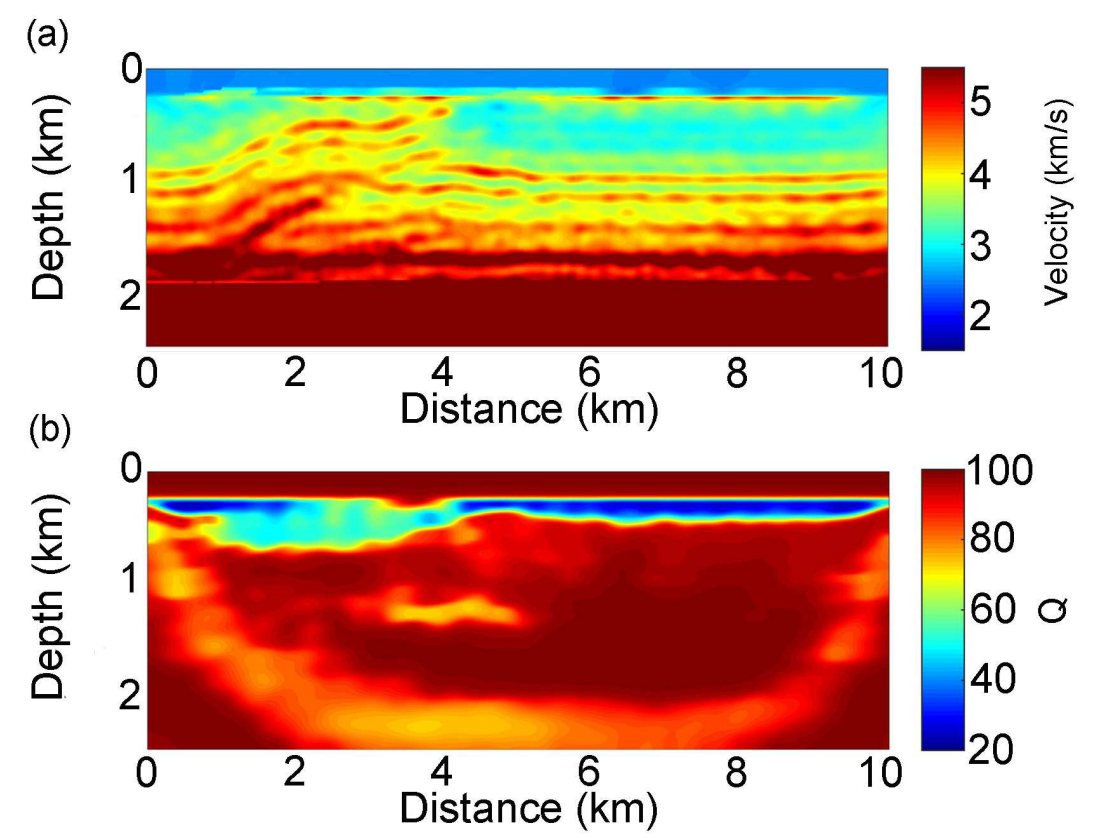

Figure 25: The sequential viscoacoustic EWI inverted velocity model (a), and $Q$ model (b) using time-domain viscoelastic data. 


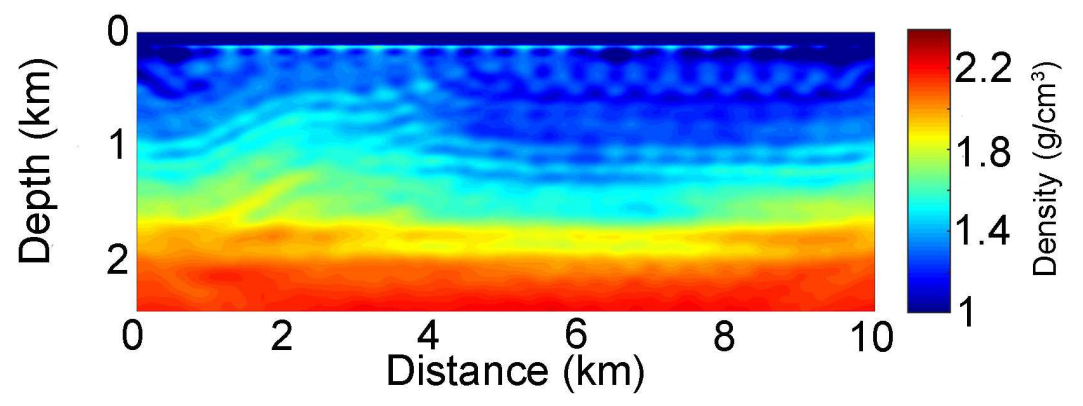

Figure 26: The inverted density model in viscoacoustic EWI. - 

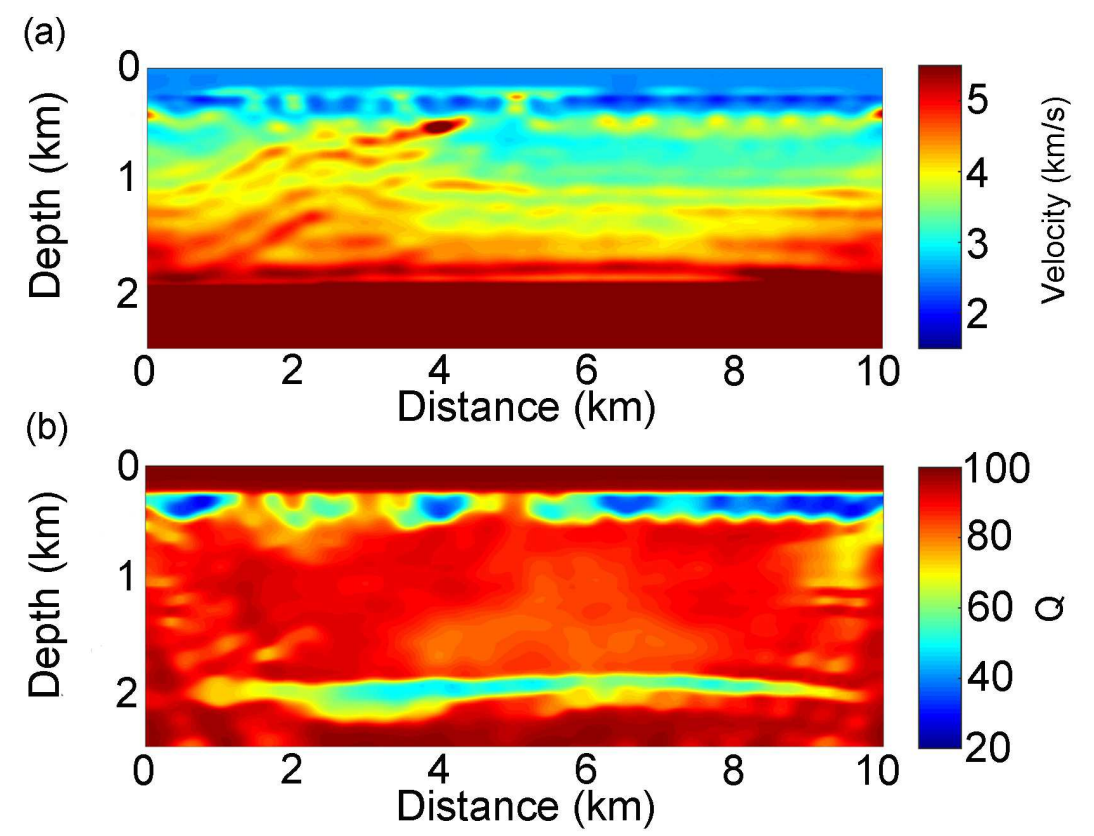

Figure 27: The sequential viscoacoustic FWI inverted velocity model (a), and $Q$ model (b) using time-domain viscoelastic data. 


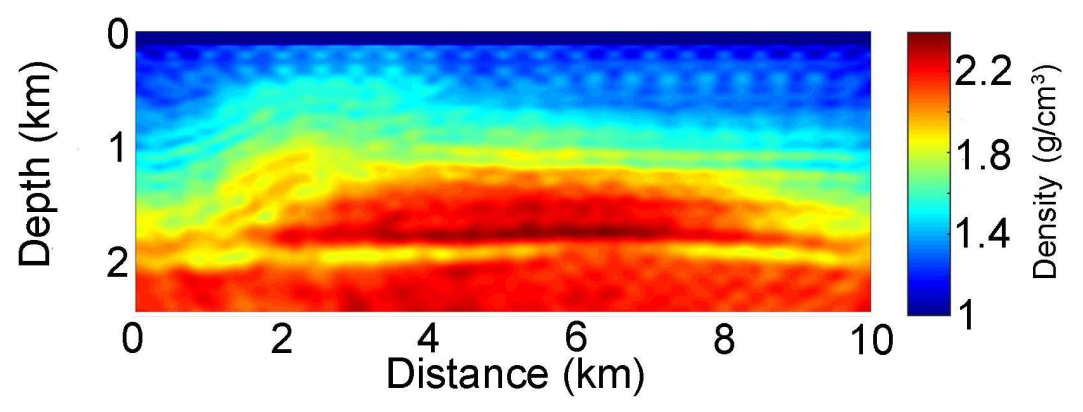

Figure 28: The inverted density model in viscoacoustic FWI. - 


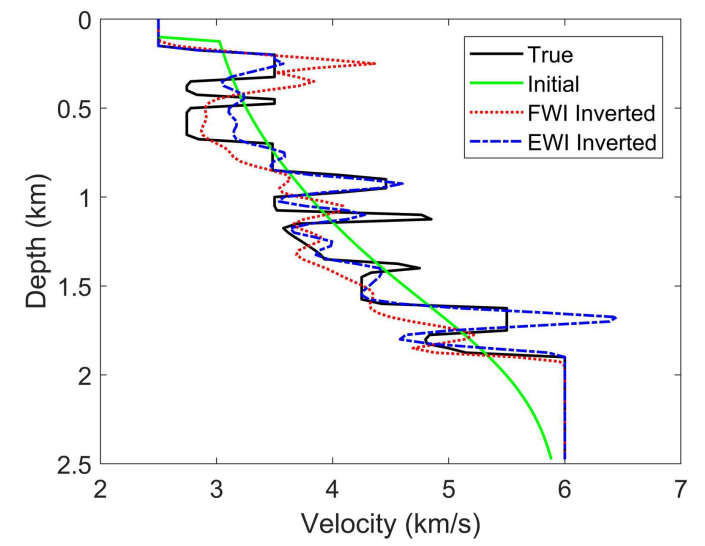

Figure 29: The vertical velocity profile at $7.5 \mathrm{~km}$ (black solid curve: true velocity, green solid curve: initial velocity, red dotted curve: sequential viscoacoustic FWI inverted velocity, blue dash-dotted curve: sequential viscoacoustic EWI inverted velocity). 